\title{
Repeated Mild Traumatic Brain Injury in Female Rats Increases Lipid Peroxidation in Neurons
}

Nathanael J. Yates ${ }^{1}$, Stephen Lydiard ${ }^{1}$, Brooke Fehily ${ }^{1}$, Gillian Weir ${ }^{2}$, Aaron Chin $^{2}$, Carole A. Bartlett ${ }^{1}$, Jacqueline Alderson ${ }^{2,3}$, Melinda Fitzgerald ${ }^{1,4,5}$

${ }^{1}$ Experimental and Regenerative Neurosciences, School of Animal Biology, ${ }^{2}$ School of Sport Science, Exercise and Health, The University of Western Australia, Crawley, 6009 Western Australia, Australia, ${ }^{3}$ Auckland University of Technology, Sports Performance Research Institute New Zealand (SPRINZ), Auckland, New Zealand. ${ }^{4}$ Curtin Health Innovation Research Institute, Curtin University and ${ }^{5}$ Perron Institute, Sarich Neuroscience Research Institute, Verdun St, Nedlands 6009 Western Australia, Australia

\section{Corresponding author:}

Melinda Fitzgerald, Prof, PhD

School of Biological Sciences, The University of Western Australia (M317)

35 Stirling Highway, Perth, WA, 6009, Australia

Tel +61 86488 2353, Fax +61 86488 1029, Email lindy.fitzgerald@uwa.edu.au 


\section{ABSTRACT}

Negative outcomes of mild traumatic brain injury (mTBI) can be exacerbated by repeated insult. Animal models of repeated closed-head mTBI provide the opportunity to define acute pathological mechanisms as the number of mTBI increases. Furthermore, little is known about the effects of mTBI impact site, and how this may affect brain function. We use a closed head, weight drop model of mTBI that allows head movement following impact, in adult female rats to determine the role of the number and location of mTBI on brain pathology and behaviour. Biomechanical assessment of two anatomically well-defined mTBI impact sites were used, anterior (bregma) and posterior (lambda). Location of the impact had no significant effect on impact forces $(450 \mathrm{~N})$, and the weight impact locations were on average $5.4 \mathrm{~mm}$ from the desired impact site. No between location vertical linear head kinematic differences were observed immediately following impact, however in the 300 milliseconds post-impact, significantly higher mean vertical head displacement and velocity were observed in the mTBI lambda trials. Breaches of the blood brain barrier were observed with three mTBI over bregma, associated with immunohistochemical indicators of damage. However, an increased incidence of hairline fractures of the skull and macroscopic haemorrhaging made bregma an unsuitable impact location to model repeated mTBI. Repeated mTBI over lambda did not cause skull fractures and were examined more comprehensively, with outcomes following one, two or three mTBI or sham, delivered at 1 day intervals, assessed on days 1 to 4 . We observe a mild behavioural phenotype, with subtle deficits in cognitive function, associated with no identifiable neuroanatomical or inflammatory changes. However, an increase in lipid peroxidation in a subset of cortical neurons following two mTBI indicates increasing oxidative damage with repeated injury in female rats, supported by increased amyloid precursor protein immunoreactivity with three mTBI. This study of acute events following closed head mTBI identifies lipid peroxidation in neurons at the same time as cognitive deficits. Our study adds 
to existing literature, providing biomechanics data and demonstrating mild cognitive disturbances associated with diffuse injury, predominantly to gray matter, acutely following repeated mTBI.findings are consistent with blood biomarkers apparent in both animal and human studies, and indicate that lipid peroxidation may be an early predictor and useful target for therapeutic intervention following concussion.

Keywords: repeated mild traumatic brain injury; oxidative stress; lipid peroxidation; closed head, weight drop model; concussion; female 


\section{INTRODUCTION}

Mild traumatic brain injury (mTBI), is relatively common, with $70-90 \%$ of all head injuries being classified as mTBI and an estimated worldwide hospitalization incidence of 100 to 300 per 100000 population (Cassidy et al. 2004). Whilst acute symptoms such as dizziness and headaches resolve within a few days to weeks, some patients exhibit chronic pathology and cognitive deficits (Kraus et al. 2007). There also appears to be cumulative effects of concussion. Retired American football athletes with a history of repeated concussion show elevated rates of mild cognitive impairment (Guskiewicz et al. 2005), with similar outcomes in athletes engaging in a variety of repeat impact sports, including boxing and soccer (Rabadi and Jordan 2001). In addition to impact force, the location and direction, of head impacts determines the likelihood of concussion (Pellman et al. 2003). However, little is known about the acute pathological mechanisms following repeated mTBI, and how these mechanisms are modified by impact location.

Common outcomes of neuronal injury are oxidative stress, neuronal death, cellular and structural disturbances and inflammation, observed in a range of animal models and patients following TBI (Werner and Engelhard 2007). Oxidative stress occurs when an increase in production of reactive oxygen and nitrogen species (ROS and RNS respectively) overcomes innate antioxidant capacity, leading to oxidative damage, and in severe instances, to activation of necrotic and apoptotic pathways (Higgins et al. 2010). Indicators of oxygen stress via ROS formation, nitrosative stresses and reduced antioxidant capacity have been consistently and extensively shown in different experimental models of TBI (Pratico et al. 2002; Hall et al. 2004; Singh et al. 2006; Cornelius et al. 2013). Given the brain's high polyunsaturated fatty acid content, after TBI oxidative stress predominantly manifests as lipid peroxidation (Hall et al. 2010). In a rat focal contusion model, there was an immediate post-traumatic burst in hydroxyl radical formation within 5 minutes $(\mathrm{min})$ of injury, which was followed by a 
progressive increase in lipid hydroperoxides (Smith et al. 1994). Acute increases in protein nitration (Ansari et al. 2008b) and DNA damage (Itoh et al. 2010) have also been observed after TBI, together with altered levels of manganese-dependent superoxide dismutase (Ansari et al. 2008a) and resultant apoptosis (Yamada et al. 2012). However, it is important to appreciate that the results from these studies of cortical impactor, lateral fluid percussion and blast injuries may not be applicable to mTBI and downstream pathological cascades may differ (Binder 1997; Dewitt et al. 2013).

The damage due to impact in mTBI is exacerbated by acceleration/deceleration forces, resulting in diffuse shear as well as compressive injury on the brain: substantial lesions, skull fracture, seizures or widespread cell death are not generally observed (Dewitt et al. 2013; Angoa-Pérez et al. 2014). Closed-head mTBI models elicit a milder phenotype than contusion injury models, without neuronal loss, and can be adapted to include head movement following impact (Kane et al. 2012; Meehan et al. 2012; Mannix et al. 2013). While lipid peroxidation and protein nitration, demonstrated by increased immunoreactivity of hydroxynonenal (HNE) and 3-nitrotyrosine (3NT) is observed following a single moderately severe closed head injury in male mice (Hall et al. 2004), together with decreases in antioxidant capacity and increases in markers of phospholipid peroxidation in a range of studies reviewed in (Signoretti et al. 2010), oxidative stress in mTBI remains to be established.

An added benefit of closed-head mTBI models is the capacity for repeated injuries to be administered to the same animal, without complications introduced by adhesions and further injury due to craniotomy. Outcomes using closed-head models of repeated mTBI suggest that there are changes in the brain that occur with repeated hits, which are not present with a single concussive impact (Fehily and Fitzgerald 2016). Repeated mTBI in mice of unspecified gender is associated with increases in the astrocytic marker GFAP in both the hippocampus and cortex following 10x injuries, assessed at 7 days, and elevated levels of phosphorylated Tau protein 
are observed in the cortex following 5x injuries assessed at 30 days (Kane et al. 2012); microglial activation was not observed. Increases in activated microglia in the corpus callosum (CC) were observed at 12 months following $5 x$ repeated mTBI delivered using a controlled cortical impact to male mice, but were not present with a single mTBI (Mouzon et al. 2014). Acute increases in microglial reactivity were also observed following 5x mTBI using a similar model (Bolton and Saatman 2014). The free-moving rotational aspect of our model is important - closed head weight-drop damage is more severe in head-restricted animals, even using the same height and weights for the mTBI (Kane et al. 2012). Repeated mTBI featuring rotational forces in mice, using similar mTBI parameters to ours (weight drop height $=0.96-1.07 \mathrm{~m}$, adjusted scaled weight for mouse : rat brain size $=257 \mathrm{~g}$ (Kane et al. 2012)) results in chronic cognitive impairments in spatial learning, but only when the interval between injuries was one week or less (Meehan et al. 2012; Mannix et al. 2013). These results suggest that whilst a single mTBI may not be overtly damaging, repeated injuries incorporating rotational acceleration and delivered within short intervals may produce more profound deficits.

Up until this point, investigations regarding the acute effects of repeated mTBI on oxidative stress in brain tissue have been somewhat limited, assessing biochemical markers in brain homogenates following two mTBI (Tavazzi et al. 2007; Vagnozzi et al. 2007). Furthermore, the majority of studies of repeated mTBI to date have utilised male animals, despite the likelihood of gender specific effects (Bazarian et al. 1999; Karr et al. 2014). The presence of oestrogen may be neuroprotective (Roof and Hall 2000) although evidence of more severe outcomes following concussions in female soccer players (Broshek et al. 2005) indicates complexities. In addition the effects of mTBI impact location (Pellman et al. 2003) on brain pathology remain relatively unaddressed. Here, we use a closed head model of repeated mTBI in female rats that incorporates head movement following impact, a 1 day inter-injury interval, using 2 different mTBI impact locations. Acute neurological, cognitive, cellular and structural 
outcomes in cortex, hippocampus and corpus callosum are assessed, and increased cell specific oxidative stress with two mTBI is demonstrated. The study design allows equal time for pathology to develop following the initial injury, in order to allow determination of the effects of further injuries on a variety of responses, some of which have been shown to be biphasic (Zhang et al. 2012). 


\section{MATERIALS AND METHODS}

\section{Study Design}

All experimentation was approved by The University of Western Australia Animal Ethics Committee (Approval Number RA/3/100/1366). Adult, female Piebald Viral Glaxo (PVG) rats (Animal Resource Centre, Murdoch, Western Australia) (160-200g) were housed in groups of 2 or 3 under standard conditions, including 12h light/dark cycles and ad libitum access to chow and water, and were habituated to conditions and handling for a minimum of 1 week before testing. Two cohorts of animals were assessed. For the first cohort comparing $3 \mathrm{x}$ mTBI delivered over bregma or lambda, animals were randomly assigned to one of six groups: Day 3 Sham $(\mathrm{N}=10)$, bregma $(\mathrm{N}=15)$, or lambda $(\mathrm{N}=15)$; or Day 4 Sham $(\mathrm{N}=8)$, bregma $(\mathrm{N}=$ 13), or lambda $(\mathrm{N}=9)$. For cohort 2 assessing the effects of repeated impacts over lambda, each animal was randomly assigned to one of 4 groups: Sham $(N=8)$, one $(1 x) \operatorname{mTBI}(N=7)$, two $(2 \mathrm{x}) \mathrm{mTBI}(\mathrm{N}=7)$, or three $(3 \mathrm{x}) \operatorname{mTBI}(\mathrm{N}=7)$.

Each animal in cohort 2 underwent 5 days of experimental testing for behavioural function. Power analyses indicated that effect sizes observed in reported studies of behavioural function following mTBI (Longhi et al. 2005; Bolouri et al. 2012) would be readily detectable using N $=7 /$ group. The first day (day 0) was a pre-training day in which each animal was assessed for Neurological Severity Score (NSS), followed by 4 sets of Morris Water maze (MWM) trials. On days 1, 2 and 3 they received either mTBI or sham treatments. Animals receiving 1x mTBI received the injury on day 1 and sham injuries on days 2 and 3. Similarly, animals receiving 2x mTBI received injuries on days 1 and 2 and Sham injury on day 3. The NSS was performed 15-20 min following each injury for each animal in order to assess acute outcomes. It is important to note that isoflurane anaesthesia was of short duration and animals recovered full consciousness within 5 min of the mTBI or sham injury. There were no differences in baseline NSS performance at day 0 compared to sham, indicating that anaesthesia did not affect 
cognitive or motor outcomes. Approximately 2 hours after the NSS each animal was tested twice for MWM performance. On the following day (day 4) there was a final NSS test followed by a probe trial of the MWM. Following the probe trial animals were euthanised with Lethabarb (500 mg/kg i.p. sodium pentobarbital, Virbac Australia), and perfused transcardially with $0.9 \%$ saline followed by $4 \%$ paraformaldehyde in $0.1 \mathrm{M}$ phosphate buffer $\mathrm{pH}$ 7.2. Brains were removed from all of the animals and post-fixed in paraformaldehyde overnight, followed by at least $72 \mathrm{~h}$ incubation in 15\% sucrose in phosphate buffered saline (PBS) solution. Before cryosectioning, the brains were hemisected mid-sagittally. The left hemisphere was sectioned in the sagittal plane and the right hemisphere was sectioned coronally. $25 \mu \mathrm{m}$ sections were collected into PBS containing $0.1 \%$ sodium azide in 24 well plates and stored at $4^{\circ} \mathrm{C}$ prior to processing. Throughout sectioning, $20 \mu \mathrm{m}$ sections were regularly collected onto Superfrost ${ }^{\circledR}$ Plus slides for Luxol Fast Blue (LFB) histochemical staining.

NSS

The assessments used were a version of the NSS (modified from: Chen et al. 1996; Stahel et al. 2000), modified to increase sensitivity for rats in terms of size of beams and balancing platforms. Performance was assessed via a 15 item checklist and was scored for the absence of a variety of reflexes and motor abilities, specifically: ability to move; to exit a $50 \mathrm{~cm}$ circle in less than 1 min; presence of righting reflex, ability to walk in a straight line; hemiplegia and monoplegia; flexion of hind-limbs when raised by the tail; startle reflex; seeking behaviour; prostration; placing reflexes for each limb; ability to stay on $5 \times 5 \mathrm{~cm}$ and $2 \times 2 \mathrm{~cm}$ platforms $30 \mathrm{~cm}$ above the ground for $1 \mathrm{~min}$; ability to balance on a $1 \mathrm{~cm}$ round beam for $1 \mathrm{~min}$ and; ability to cross $3 \mathrm{~cm}, 2 \mathrm{~cm}$ and $1 \mathrm{~cm}$ beams $30 \mathrm{~cm}$ above the floor without foot faults. The scoring system allows for a maximum possible score of 26; data are non-linear ranking scores where a score of approximately 5 equates to behaviour of normal un-injured animals. 
The apparatus was a $1.65 \mathrm{~m}$ diameter pool (HVS Image) filled to a depth of $30 \mathrm{~cm}$ with water heated to $24-26^{\circ} \mathrm{C}$ with an aquarium heater, and made opaque by the addition of white poster paint (Crayola). The pool edge was marked with the points of a compass so that the swimming area was divided into quadrants (NE, NW, SE and SW). Testing was conducted according to established procedures (Morris 1984). Briefly, during the pre-training days and treatment days (days 0 to 3 ) the animals were trained to find a $10 \mathrm{~cm}$ round platform placed in the $S W$ quadrant that was covered by 1-2 $\mathrm{cm}$ of water, thereby obscuring it from view. During pre-training and subsequent testing animals were placed in the water facing the wall of the pool and were allowed to swim until they found the hidden platform, or until 2 min passed, whichever came first. If 2 min passed without animals locating the platform, they were guided to its location. After reaching the platform the animals were allowed to stay on the platform for $15 \mathrm{~s}$, after which they were dried and placed under a $50 \mathrm{~W}$ heating lamp. Animals were tested in sets of 4 trials in pseudorandom order, with one trial starting in each quadrant. A webcam (C270 Logitech) was mounted on the ceiling and connected to a laptop allowing for video recording (iSpy, www.ispyconnect.com/) of the tests. An interval of 10-15 min was maintained between each set of trials for each animal. On day 4 the probe trial test was performed, where the platform was removed and the rats were videoed for $90 \mathrm{~s}$ before being removed from the water. The video was analysed using video tracking software ANY-Maze (Stoelting Co.) and outcome measures of: time in each quadrant; distance from platform; distance travelled and time to reach platform were assessed. Investigators conducting the scoring were blinded to the number of mTBI an animal had received.

\section{mTBI Procedure}

mTBI was delivered using a custom built weight-drop device (Northeast Biomedical Inc. MA, USA) (Figure 1A), largely as described in Kane et al. (2012). A $250 \mathrm{~g}$ (cross-sectional area = $5 \mathrm{~mm}^{2}$ ) weight was chosen for the current study, as pilot testing revealed that this was the 
heaviest weight that could be used repeatedly to deliver an impact over lambda on the same PVG female rats without causing skull fracture or haemorrhage. In brief: animals were anaesthetised with $4.5 \%$ Isoflurane in $4 \mathrm{~L} / \mathrm{min}$ oxygen, maintained throughout the procedure via a nose cone. The animal was placed on a delicate task wiper (Kimwipes, Kimberley-Clark Inc.) that was secured over a platform with a hole in the centre (Figure 1B). The animal's head was placed directly under the guide tube such that the impact site was mid-sagittal and 2-3 mm anterior to the front of the ears, which corresponded to the location of lambda (Figure 1C) on the skull. An alternative impact location over bregma was also employed for comparative purposes (Figure 1D). A $250 \mathrm{~g}$ weight was released from a height of $1 \mathrm{~m}$ onto the impact location. At impact, the rat's head penetrated the Kimwipe, resulting in movement of the head and a $180^{\circ}$ vertical rotation of the animal's body as it fell $15 \mathrm{~cm}$ onto soft foam padding. The fall of the animal onto the foam bed incorporates rotational forces into the model, thereby increasing clinical applicability. The weight was restrained from multiple impacts with the head, by use of a restraining lead attached to the top of the weight. Immediately following the impact analgesia was administered (4 mg/kg carprofen subcutaneously, Carprieve, Norbrook Laboratories Australia), and the rat was placed on a $37^{\circ} \mathrm{C}$ heating pad to recover. The length of time the animal remained unconscious following mTBI or sham was recorded, and was typically less than $5 \mathrm{~min}$. The location of the impact on the skull was visualised using white paint placed on the weight before release, and the site of impact was recorded. Sham injuries were identical, including anaesthesia, except that the weight was not released.

\section{Biomechanical assessment}

To test the reliability of the weight-drop device apparatus protocols, and to establish baseline biomechanical measures, a single repeated mTBI data collection was performed using a recently euthanised adult female PVG rat. A fully synchronised 6-camera Vicon MX near infrared motion capture system (Vicon, Oxford Metrics Ltd., UK) and one Vicon Bonita grey-scale 
video camera $(250 \mathrm{~Hz})$ were used to record the relative motion of the weight drop and the resulting post-impact movement of the animal via spherical retroreflective three-dimensional (3D) markers (Figure 1A). Permanent $8 \mathrm{~mm}$ static markers affixed in each corner or the apparatus baseplate established the reference global coordinate system. Retroreflective tape was placed around the impact nodule of the weight mass acting as a marker to facilitate realtime location tracking and subsequent direct kinematic calculations. To avoid excessive skin and soft tissue movement artefact, permanent adhesive was used to affix a single retroreflective marker anterior to the nasal bone, and a baseplate comprising a four marker $(4 \times 4 \mathrm{~mm})$ cluster was affixed to the flat part of the bone between the Angle and Ramus of the lower mandible which was exposed for fixation by scraping away the overlying muscle (Figure 1B). Lambda and bregma sites were identified in the capture reconstruction volume using a single $4 \mathrm{~mm}$ marker during a static calibration trial and the positions virtually stored relative to a technical coordinate system created by the four marker cluster wand. The marker was removed during all impact trials, with the lambda and bregma locations reconstructed in dynamic impact trials using the stored cluster reference coordinates from the static calibration trial. Drop weight and animal affixed trajectories were labelled and processed using a customised bodybuilder model in Vicon Nexus software. Following the static calibration trials a total of 8 repeated mTBI trials were performed (4 lambda followed by 4 bregma). Following each mTBI trial the laboratory technician manually repositioned the rat following standardised procedures for impacting the identified lambda and bregma sites. Following visual inspection of the raw trajectory data, 3 trials with superior data quality from each target site were selected for modelling and subsequent analysis.

\section{Evans Blue assessments of blood brain barrier integrity}

For cohort 1, a subset of animals were injected via the tail vein with $2 \%$ (w/v) Evans Blue (Sigma, E-2129_10G), either one hour prior to the last of 3x mTBI on day 3 (pre-mTBI group), 
or 24 hours following the last of $3 x$ mTBI on day 4 ( $24 \mathrm{hrs}$ group) $(\mathrm{N}=4-5 /$ group). Animals were euthanized with Lethabarb $1 \mathrm{~h}$ after Evans Blue injection $(500 \mathrm{mg} / \mathrm{kg}$ i.p. sodium pentobarbital, Virbac Australia), and perfused transcardially with $0.9 \%$ saline followed by $4 \%$ paraformaldehyde in $0.1 \mathrm{M}$ phosphate buffer $\mathrm{pH} 7.2$. Brains were removed from all of the animals and post-fixed in paraformaldehyde overnight, followed by 72 hours incubation in $15 \%$ sucrose in phosphate buffered saline (PBS) solution. Brains were cryosectioned coronally; $18 \mu \mathrm{m}$ sections were collected onto Superfrost ${ }^{\circledR}$ Plus slides for immediate visualisation, to minimise leaching of Evans Blue from the tissue. Presence of Evans Blue in cortex in 8 entire coronal brain sections per animal were assessed by a single investigator blinded to sample identity, and each individual blood brain barrier breach was scored as,+++ , or +++ according to the absolute size of the breach viewed at 20x magnification. Scores were assigned a number of 1,2 , or 3 respectively and numbers summed to generate a blood brain barrier breach score. Note that autofluorescence in the sections precluded automated image analysis, which would erroneously capture false positive pixels, thereby masking changes with injury. Careful examination of the sections via fluorescence microscopy, where autofluorescence can be differentiated, was required to score the degree of breach within each part of each section. A subset of representative images for each breach score received full quantitative analysis of the breach area as a percent of cortical area, by manually tracing around images, with reference to sections viewed by microscopy. The mean percentage areas of the cortical regions for individual breaches in sections that were positive for Evans Blue were $0.8 \%, 1.9 \%$ and $3.1 \%$ for,+++ and +++ respectively. Additional immunohistochemistry analyses were conducted on sections from cohort 1 , as described for cohort $2(\mathrm{~N}=8-9 /$ group total $)$.

\section{Immunohistochemistry}

All immunochemical analyses were performed on free-floating sections in multiwell plates from all experimental animals using established procedures as follows. Sections were washed 
in PBS (pH 7.2 - 7.4) for $2 \times 5$ min followed by PBS containing 0.2\% Triton-X 100 and 5\% normal donkey serum (blocking serum) for $10 \mathrm{~min}$. Sections were then incubated overnight at $4^{\circ} \mathrm{C}$ in blocking serum containing primary antibodies detecting: oxidative stress indicators, 4hydoxynonenal (HNE) (rabbit 1:500, Alpha Diagnostics); 8-hydroxy-2'-deoxyguanosine (8OHDG) (mouse 1:500, Abcam); 3-nitrotyrosine (3NT) (1:500; Abcam, Cambridge, UK), antioxidant enzyme Manganese Superoxide Dismutase (MnSOD) (rabbit 1:500, Enzo Life Sci); programmed cell death indicator cleaved Caspase 3 (rabbit 1:500, Cell Signalling); Anti Alzheimer precursor protein antibody (AlzPP) (mouse, 1:500, Merck Millipore (MAB348)_ note that this antibody is frequently referred to as anti-APP in the literature (Buchele et al. 2016); resident microglia/macrophages, IBA1 (goat1:1000, Abcam) (Ito et al. 1998); oligodendrocyte precursor cells (OPCs), Olig2 (goat 1:500, Abcam) (Gao et al. 2006) together with NG2 (rabbit 1:500, Merck Millipore); mature oligodendrocytes, CC1 (mouse, 1:500, Calbiochem) (Fuss et al. 2000); astrocytic glial fibrillary acid protein (GFAP) (goat 1:1000, Abcam); neurons, $\beta$ III-tubulin (mouse 1:1000, Covance) or NeuN (mouse 1:500, Chemicon)(Mullen et al. 1992); paranodes, Caspr (rabbit 1:500, Abcam); myelin basic protein (goat, 1:500, Santa Cruz). The following day sections were washed with PBS ( 3 x 5 min), then incubated for 2 hours at room temperature with Hoechst nuclear stain $(0.5 \mu \mathrm{g} / \mathrm{ml}$, Invitrogen) and appropriate species specific secondary antibodies AlexaFluor ${ }^{\circledR} 488,555$ or $647,1: 500$ Invitrogen). Following 2 x 5 min PBS washes sections were mounted onto glass slides, air dried, and cover-slipped with Fluoromount-G (Southern Biotech). Immunohistochemistry on sections from all animals and across all groups were conducted at the same time to avoid interrun variability.

LFB staining was performed on sections collected onto Superfrost ${ }^{\circledR}$ Plus slides, by first dehydrating sections in an ascending alcohol series (70\% to $100 \%$ ethanol), defatting with xylene and rehydrating through descending concentrations of alcohol (100\% to $70 \%$ ethanol). 
Sections were then placed in $0.1 \%$ LFB solution in $95 \%$ ethyl alcohol and $0.5 \%$ glacial acetic temperature, rinsed with $95 \%$ ethanol for $30 \mathrm{~s}$, rinsed with PBS, differentiated in $0.05 \%$ lithium carbonate for $30 \mathrm{~s}$, differentiated in $70 \%$ ethanol for $30 \mathrm{~s}$, rinsed in PBS and the differentiation process repeated once more. The slides were subsequently stained with Cresyl violet $(0.1 \%$ in $\mathrm{dH}_{2} \mathrm{O}$ and $0.01 \%$ sodium acetate, $\mathrm{pH} 3.4$ ), at $60{ }^{\circ} \mathrm{C}$ for $10 \mathrm{~min}$, rinsed in PBS, differentiated in $95 \%$ ethanol for 5 min, dehydrated in $100 \%$ ethanol, cleared in xylene and cover-slipped in Entellan ${ }^{\circledR}$ New (Merck).

FluoroJade C staining was performed on $25 \mu \mathrm{m}$ sections mounted onto Superfrost ${ }^{\circledR}$ Plus slides. Slides were air-dried then incubated at $50-55^{\circ} \mathrm{C}$ for $30 \mathrm{~min}$. This was followed by brief immersion in PBS, immersion in $80 \%$ ethanol with $1 \% \mathrm{NaOH}$ for $5 \mathrm{~min}$, a rinse for 2 min in $70 \%$ ethanol, then $2 \mathrm{~min}$ in distilled water. They were then incubated in $0.06 \%$ potassium permanganate for $10 \mathrm{~min}$, and rinsed for $2 \mathrm{~min}$ in distilled water. Slides were then incubated in $0.0001 \%$ FluoroJade C (Merk Millipore) in $0.1 \%$ Acetic acid for $10 \mathrm{~min}$, rinsed in distilled water 3 x 1 min, air-dried, cleared in xylene and cover-slipped in Entellan ${ }^{\circledR}$ New (Merck).

\section{Microscopy and Image Analysis}

Brain regions were defined for imaging as follows. For sagittal sections: posterior CC was defined as the splenium of the $\mathrm{CC}$; middle $\mathrm{CC}$ was the most anterior position above the hippocampus in the section; anterior was the genu of the CC. Cortex: posterior cortex was defined as the most posterior cortex surface in the section; middle cortex was the dorsal cortex above the hippocampus; anterior cortex, the dorsal surface of the cortex above the anterior CC. Division of the regions is illustrated in Figure 4A. Further regions of analysis were defined relative to the corpus callosum anatomy. Coronal sections anterior to the genu of the corpus callosum were defined as anterior, sections at the level of the genu as middle, and at the level 
of the splenium as posterior. Note that these defined regions approximately match the regions defined in the sagittal plane as anterior, middle, and posterior.

Imaging for quantification was performed using a Nikon, TI-E Inverted Microscope, controlled by NIS elements version 4.0 software for multi-channel images; or a Leitz Diaplan microscope with an Olympus DP70 camera controlled by DP controller (version 2.1.1.183) for single channel images. Images for co-localisation analysis of HNE used a confocal Nikon c2 mounted on an upright Ni-E microscope, controlled by NIS elements 4.3 software.

Cell counts and immunointensity analyses were performed on a single image per animal, normalised for area, in accordance with previously published procedures (Szymanski et al. 2013; O'Hare Doig et al. 2014). The only exception was HNE cell counts, which were conducted on photomontages of the entire cortex of a single section from each animal. Given the absence of a defined injury site and in order to ensure a consistent location and to minimise variability, sagittal sections closest to the midline were selected for quantification, and coronal sections for analysis were taken at the level of the genu of the corpus callosum. Note that a stereological approach was not considered necessary, as quantification of total cell numbers in specific brain regions was not the aim of the study. Montaging of images was conducted using Photoshop CC (2014.2.2 release, Adobe) or directly in NIS software. For each outcome measure where intensity of immunoreactivity was analysed, sections were visualized and imaged in a single session: images were captured at constant exposure settings across all groups. Image analysis was conducted on a single, central visual slice using ImageJ/Fiji analysis software, setting constant arbitrary threshold intensities for all images in an analysis and determining the mean intensity and area above the threshold intensity and the mean intensity of fluorescence of the image, as per established procedures (O'Hare Doig et al. 2014). All images used a single image size for each set of data, cropping images as necessary to only include the regions of interest. All quantifications of cell numbers and immunointensity were 
conducted by investigators blinded to sample identity. Control sections in which the primary antibody was omitted were included for all immunohistochemistry assessments and minimal staining was observed (not shown).

HNE+ cells were defined based upon strong somatic staining of the cell, together with a clearly delineated profile, visible nucleus, and fluorescence well above that of background surrounding cells. CC thickness was determined using transverse sections stained for LFB and Cresyl violet. Thickness was determined by measuring the dorso-ventral depth of LFB+ axons at the midline of the CC. Total width, including the external and internal capsules of the CC, was also measured. Node-paranode analyses were conducted using our previously published techniques (O'Hare Doig et al. 2014). In brief, transverse sections of the posterior CC nearest the mTBI impact site were imaged at 40x optical magnification to detect Hoechst, $\beta$ III-tubulin and Caspr in $6 \mu \mathrm{m}$ z-stacks at $0.5 \mu \mathrm{m}$ optical thickness, and deconvolved using NIS software. A region on the dorsal-medial surface of the $\mathrm{CC}$ was examined and dimensions of all clearly defined Caspr+ node-paranode complexes therein were quantified throughout the z-stack (counting 22 to 58 node/paranode complexes per animal). Note that $\beta$ III-tubulin immunoreactivity is not shown in representative images, to aid visualisation of paranodes. Investigators analysing images were blinded to group identity.

\section{Statistical Analysis}

A conservative method of statistical analysis was employed, where initial analysis did not assume ordinal effects. As such, it was not assumed that $3 \mathrm{x}$ mTBI would have a larger effect than $2 \mathrm{x}$ mTBI and so on. If there were significant differences at the omnibus level of assessment, post-hoc testing was performed. Where appropriate, data were assessed for correlations relative to the number of mTBI. Categorical data were analysed using Chi-squared testing, normally distributed parametric data was analysed using single or repeated measures two-way ANOVAs as appropriate, and non-normally distributed parametric data using 
Kruskal-Wallis rank sum test. Post-hoc testing compared mTBI groups to Sham for the specific day of analysis, and used Holm-Bonferroni alpha level corrections for multiple comparisons. All statistics was performed using the statistical program R (version 3.2.2) in R Studio (version 0.99.482) with packages ez (version 4.2-2) and plyr (version 1.8.3). Graphs were made using GraphPad Prism 7 (version 7.02, GraphPad Software) and images were edited and analysed with ImageJ (version 1.5e). 


\section{RESULTS}

\section{Biomechanical Analysis}

We assessed the biomechanical aspects of our closed-head mTBI model in 2 impact site locations. The reliability of the weight-drop apparatus as the weight exited the guide tube across all $6 \mathrm{mTBI}$ trials is evidenced by a mean deviation of $+/-0.1 \mathrm{~mm}$ from the global origin of laboratory based coordinate system. Mean drop-weight velocity directly recorded $0.004 \mathrm{~s}$ (1 frame) prior to impact was $3.6 \pm 0.19 \mathrm{~m} . \mathrm{s}^{-1}$ (bregma $3.6 \pm 0.3$; lambda $3.6 \pm 0.01$ ). Given the known mass of $250 \mathrm{~g}$, the mean pre-impact kinetic energy across all mTBI trials was $1.63 \pm$ $0.2 \mathrm{~J}$ (bregma 1.6 \pm 0.28 ; lambda 1.6 \pm 0.01 ). With mean impact duration of $0.004 \mathrm{~s}$, the directly calculated mean impact force using the direct weight velocity was $450 \pm 24 \mathrm{~N}$ (bregma $450 \pm$ $1 \mathrm{~N}$; lambda $451 \pm 38 \mathrm{~N}$ ). These impact forces are lower than those reported in similar studies however the present study directly measured the input parameters as opposed to using estimates based on conservation of energy equations which do not take into account the effect of air resistance and guide tube friction on the drop weight. For example, when estimating weight velocity at impact using the known weight and height of the drop distance ( $1 \mathrm{~m}$ guide tube plus striking distance of $0.05 \mathrm{~m}$ ) as per Mychasiuk et al., (2016), the estimated weight velocity of the present study should be $4.5 \mathrm{~m} / \mathrm{s}$, approximately $0.94 \mathrm{~m} / \mathrm{s}$ higher than the directly recorded value. The lower weight velocity directly recorded prior to impact resulted in a net reduction of $\approx 110 \mathrm{~N}$ of impact force when compared with estimations using standard energy equations applied to falling objects. Researchers relying on such assumptions when employing similar weight-drop apparatus should be aware that where direct estimates of the input parameters into energy equations are not available, there will likely be an over estimation of drop-weight velocities and resultant impact forces.

The distance recorded in the static calibration trial between the ground-truth (technician identified) bregma and lambda impact site was $9.6 \mathrm{~mm}$ with $14.1 \pm 2.7 \mathrm{~mm}$ recorded as the 
mean distance separating the mTBI bregma $(\mathrm{N}=3)$ and lambda $(\mathrm{N}=3)$ trials. Across the $6 \mathrm{x}$ mTBI trials the overall mean 2D (X\&Y) Euclidean distance between the ground-truth bregma and lambda locations and the directly recorded impact locations was $5.4 \pm 2.3 \mathrm{~mm}$. Greater differences were recorded in the lambda mTBI impacts $(6.9 \pm 2.0 \mathrm{~mm})$ where the location tended to be anterolateral (left) to the ground-truth location (Figure 1C). Bregma mTBI impacts $(4.0 \pm 1.6 \mathrm{~mm})$ tended to be anterior to the ground-truth site with no obvious lateral bias (Figure 1D). The minimal deviation of the weight-drop on exit of the guide tube indicates that the discrepancies between the impact and ground-truth sites can be attributed to the difficulty in manually positioning a pliable animal on an unstable and delicate surface (task wiper) during initial positioning.

Immediate $(0.004 \mathrm{~s})$ post-impact mean head vertical linear velocities were similar between target strike locations bregma: $1.46 \pm 0.35 \mathrm{~m} / \mathrm{s}$; lambda: $1.37 \pm 0.21 \mathrm{~m} / \mathrm{s})$. Longer post-impact examination encompassing the fall period $(0.32 \mathrm{~s})$ revealed small but statistically significantly higher mean head vertical drop displacement $(\mathrm{p}=0.03)$ and velocity $(\mathrm{p}=0.02)$ following lambda trials $(7.2 \pm 0.51 \mathrm{~cm} ; 2.15 \pm 0.11 \mathrm{~m} / \mathrm{s})$ compared with bregma trials $(5.5 \pm 0.06 \mathrm{~cm}$; $1.71 \pm 0.01 \mathrm{~m} / \mathrm{s}$ ). Marker occlusion due to the trajectory of the animal and the broken task wiper resulted in unreliable data for quantifying head angular velocity/acceleration during this time period, however qualitative inspection of the video footage confirms a substantially higher rate of animal angular rotation following lambda strikes, whereby a full $180^{\circ}$ rotation of the animal was achieved in a mean $0.16 \pm 0.02 \mathrm{~s}$ compared with the bregma time of $0.28 \pm 0.01 \mathrm{~s}$. (supplementary material).

\section{General Observations and Comparisons Between 3x mTBI Over Bregma and Lambda}

There were no indications of respiratory depression, seizure or any obvious behavioural alterations following the administered mTBI or Sham injuries in female PVG rats. There were no differences in time to wake from anaesthesia for any day or injury group (ANOVA, all $\mathrm{p}$ > 
0.05, data not shown), emphasising the mild nature of the injury delivered. For cohort 1 , upon euthanasia on days 3 or 4 , thin long "hairline fractures" were observed following $3 \mathrm{x}$ mTBI over bregma in $25.8 \%$ of animals, with macroscopically evident haemorrhaging in $45.1 \%$ of animals. There was no evidence of skull damage or haemorrhage for any animals experiencing 3x mTBI over lambda. For cohort 2, upon euthanasia on day 4, there were no skull fractures, brain haemorrhaging or subdural bleeding, with only superficial bruising on the skull surface.

Blood brain barrier breaches were detected as increased areas of Evans Blue fluorescence, with regard to the limitations of the method (Saunders et al. 2015). Increased blood brain barrier integrity scores indicative of breaches in the barrier in the cortex were detected on day 3 (day $3, \mathrm{~F}(2,10)=5.83, \mathrm{p} \leq 0.05 ;$ day $4, \mathrm{~F}(2,11)=0.14, \mathrm{p}=0.87)$ and post hoc analysis revealed increases following 3x mTBI over bregma relative to sham controls when assessed on day 3 , immediately following injury $(\mathrm{p} \leq 0.05$, Figure $2 \mathrm{~A}$, representative images of scores are in Figure 2B).

Immunohistochemistry was used to detect the presence of HNE, an indicator of phospholipid peroxidation (Schneider et al. 2008) in coronal sections of the brain. HNE immunohistochemical analysis revealed the presence of large multipolar HNE+ cells with large centrally located nuclei in the cortex and these were quantified. There were differences in the number of HNE+ cells in cortex following 3x mTBI dependant on impact location on day 3 but not day 4 (day $3, \mathrm{~F}(2,20)=4.44, \mathrm{p} \leq 0.05$; day $4, \mathrm{~F}(2,21)=1.46, \mathrm{p}=0.26)$. Post hoc analysis revealed a significant increase in the number of HNE+ cells following 3x mTBI over bregma relative to sham on day $3(\mathrm{p} \leq 0.05$, Figures $2 \mathrm{C}, \mathrm{D})$.

IBA1+ cells in regions of posterior, middle and anterior cortex were separately quantified and differences with impact location were observed on days 3 and 4 (day 3 , posterior $F(2,21)=$ $0.03, \mathrm{p}=0.97$; middle $\mathrm{F}(2,22)=0.22, \mathrm{p}=0.81$; anterior $\mathrm{F}(2,12)=13.75, \mathrm{p} \leq 0.001$ : day 4 , posterior $\mathrm{F}(2,14)=1.77, \mathrm{p}=0.21$; middle $\mathrm{F}(2,19)=3.04, \mathrm{p}=0.07$; anterior $\mathrm{F}(2,15)=3.86$, 
$\mathrm{p} \leq 0.05)$. Post hoc analysis revealed a significant reduction in the number of IBA1+ cells following $3 \mathrm{x}$ mTBI on day 3 over lambda and bregma in anterior cortex relative to sham $(\mathrm{p} \leq$ 0.001 and $\leq 0.01$ respectively) and there was a significant reduction for day 4 bregma impacts relative to sham $(\mathrm{p} \leq 0.05)$ (Figure $2 \mathrm{E}, \mathrm{F})$.

Myelin basic protein (MBP) immunoreactivity in regions of posterior, middle and anterior cortex as well as the underlying $\mathrm{CC}$ were quantified on day 3 and differences with impact location were observed in both cortical regions and the CC with 3x mTBI. Specifically, regionspecific increases in both cortex MBP immunointensity (posterior $\mathrm{F}(2,21)=2.1, \mathrm{p}=0.15$; middle $F(2,23)=2.78, p=0.083$; anterior $F(2,18)=4.043, p \leq 0.05)$ (Figure 2G) and the MBP immunopositive area as a ratio of total cortex area $($ posterior $\mathrm{F}(2,21)=1.94, \mathrm{p}=0.17$; middle $\mathrm{F}(2,23)=4.18, \mathrm{p} \leq 0.05$; anterior $\mathrm{F}(2,18)=4.22, \mathrm{p} \leq 0.05$, data not shown) were observed. Post hoc analysis revealed a significant increase in MBP immunoreactivity following $3 \mathrm{x}$ mTBI over bregma in anterior cortex relative to sham controls $(\mathrm{p} \leq 0.05)$ and area of immunopositivity for anterior and middle cortex (both $\mathrm{p} \leq 0.05$; Figures $2 \mathrm{G}, \mathrm{H}$ ), likely indicative of myelin disruption resulting in increased immunogenicity (Kozlowski et al. 2008). Importantly region-specific increases in MBP immunoreactivity were observed in the CC with $3 \mathrm{x}$ mTBI $($ posterior $\mathrm{F}(2,22)=2.92, \mathrm{p}=0.075$; middle $\mathrm{F}(2,23)=4.079, \mathrm{p} \leq 0.05$; anterior $\mathrm{F}(2$, $16)=1.51, \mathrm{p}=0.25)$, with post hoc analyses confirming increases between sham and bregma groups in the middle corpus callosum $(\mathrm{p} \leq 0.05)$.

\section{Behavioural Outcomes following increasing numbers of mTBI over lambda}

Given the increased number of animals with hairline skull fractures when $3 x$ mTBI were administered over bregma, further analysis was confined to animals receiving mTBI over lambda. Behavioural outcomes were assessed in experimental groups that had received, sham, 1x, 2x or 3x mTBI over lambda. There were no significant differences in total NSS following single or repeated mTBI on any day of testing (Kruskal-Wallis rank-sum, Figure 3A), although 
there was a significant increase in the proportion of foot faults on the $1 \mathrm{~cm}$ beam with $1 \mathrm{x} \mathrm{mTBI}$, when all data were pooled on day 1 (Figure 3B). There were also no differences in recall of the platform location in the MWM between shams, single or repeated mTBI groups on days 1,2 or 3 (Figure 3C). Significant differences on days 2 and 3 were only observed between sets 1 and 2 for individual groups, reflecting learning within the day (Day 2: $\mathrm{F}(1,26)=10.19, \mathrm{p} \leq$ 0.01; Day 3: $\mathrm{F}(1,25)=13.09, \mathrm{p} \leq 0.01)$. There was no evidence of changes due to the mTBI injuries, as there were no main effects or interactions with injury group on any given day on days 1 to 3 (all $p>0.05$ ). For the MWM probe trial on the day of euthanasia (day 4 ) there were significant differences in recall of the platform location as a consequence of repeated mTBI. The time spent in the quadrant opposite the previous platform location (Figure 3D, F(3, 25) = $3.54, \mathrm{p} \leq 0.05$ ), in the quadrant that previously contained the platform (Figure 3E, $\mathrm{F}(3,25)=$ $3.31, \mathrm{p} \leq 0.05)$ and the mean distance from the platform differed between groups (Figure $3 \mathrm{~F}$, $\mathrm{F}(3,25)=3.09, \mathrm{p} \leq 0.05)$. Post-hoc analysis revealed that in each of these measures the $2 \mathrm{x}$ mTBI group was significantly different from the sham group $(\mathrm{p} \leq 0.05)$, whereas the $1 \mathrm{x}$ and $3 \mathrm{x}$ mTBI groups were not different from sham $(\mathrm{p}>0.05)$. There was also a moderate positive correlation between number of mTBI and mean distance from the platform (Figure 3G, $\mathrm{R}=$ $0.35, \mathrm{p} \leq 0.05)$. There were no differences in the mean swimming speed between groups $(\mathrm{F}(3,25)=0.392, \mathrm{p}=0.760$, data not shown $)$, indicating that differences during the probe trial were not due to motor impairment or motivation impairment.

\section{Indicators of Oxidative Stress and Cellular Death}

HNE+ cells were distributed throughout the cortex but not the CC, so the numbers of HNE+ cells were quantified in entire mid-sagittal sections of the cortex (Figure 4A). HNE+ cell numbers did not follow a normal distribution, therefore Kruskal-Wallis rank sum tests were used to determine statistically significant differences. The total numbers of HNE+ cells normalised for area were different between mTBI groups for both the middle cortex $(\mathrm{H}=8.45$, 
df $=3, p \leq 0.05)$ and total cortex $(H=10.50, \mathrm{df}=3, \mathrm{p} \leq 0.05)$, but not for the anterior $(\mathrm{H}=$ 2.36, $\mathrm{df}=3, \mathrm{p}>0.05)$ or posterior cortex $(\mathrm{H}=6.48, \mathrm{df}=3, \mathrm{p}>0.05)$ (Figure 4B). Post-hoc comparisons revealed a significant increase in $\mathrm{HNE}+$ cell numbers in animals receiving $2 \mathrm{x}$ mTBI compared to sham injured animals, for both the middle cortex and total cortex $(\mathrm{p} \leq 0.05)$; outcomes following $1 \mathrm{x}$ mTBI or $3 \mathrm{x}$ mTBI were not different to sham injured animals $(\mathrm{p}>$ $0.05)$.

The identity of HNE+ cells was determined immunohistochemically throughout the cortex using individual visual slices within the $\mathrm{z}$ plane collected by confocal microscopy. Cells that were immunoreactive for $\mathrm{HNE}$ were not immunoreactive for NG2, indicating that HNE+ cells were not OPCs or pericytes (Figure 4C). Similarly, HNE+ cells were not immunoreactive for $\mathrm{CC} 1$ or olig2, indicating that these cells were not more mature oligodendrocytes (Figure 4D) (Psachoulia et al. 2009; Payne et al. 2013). HNE+ cells were also not immunoreactive for markers of microglia (IBA1, Figure 4D) or astrocytes (GFAP, Figure 4F), but HNE was exclusively present in cells immunopositive for the neuronal marker NeuN (Figure 4F).

Because of the increase of lipid peroxidation with repeated mTBI we examined brain tissue for others signs of brain damage or oxidative stress (Figure 5). Immunoreactivity for 8OHDG, an indicator of oxidative damage to DNA (Valavanidis et al. 2009), was present in large numbers of cells throughout the cortex, but not in the CC. There were no differences in 8OHDG fluorescence intensity following single or repeated mTBI in any region examined (ANOVA, all $\mathrm{p}>0.05$, Figure 5A). HNE immunoreactivity co-localised with $8 \mathrm{OHDG}$ in a small subset of cells, which had a cell profile reminiscent of $\mathrm{NeuN}+$ cells (data not shown). MnSOD immunoreactivity increases in primary and secondary neurodegeneration (Fitzgerald et al. 2010), however examination of brain sections did not reveal any pockets of MnSOD immunoreactivity or distinctly MnSOD+ cells in the cortex or CC. However in the hippocampus there were many cells that appeared distinctly MnSOD positive (Figure 5B). 
However quantification of the number of MnSOD+ cells in the dentate gyrus of the hippocampus did not reveal differences with single or repeated mTBI (Kruskal-Wallis, p > 0.05). Immunoreactivity of 3-NT, an indicator of protein nitration, was not above background in tissue sections from either sham or mTBI groups, in any region examined (data not shown). Markers of cellular stress and degeneration were also assessed. Quantification of cells positive for the apoptosis marker Caspase 3, in the dentate gyrus of the hippocampus and in the posterior, middle, and anterior $\mathrm{CC}$, did not reveal any changes with single or repeated $\mathrm{mTBI}$ (ANOVA, all $p>0.05$, Figure 5C). In the hippocampus, Caspase 3 appeared to often be present in cells which were GFAPt, but differences with mTBI were not apparent. The number of Caspase 3 immunopositive cells likely reflects normal cortical cell turnover in adult rat and are in line with estimated densities in sham injured rats reported in similar studies (Abdul-Muneer et al. 2013).FluoroJade C staining, a specific marker of neuronal degeneration, was not observed in any brain region, despite being present in positive control tissue of degenerating optic nerve (data not shown). In contrast, AlzPP immunoreactivity in the middle cortex was present in a perinuclear distribution in more cells and at a higher intensity when animals had undergone repeated mTBI than single or sham injury (Figure $5 \mathrm{D} \underline{\mathrm{CC}}$ ). Similar outcomes were observed in posterior cortex. These patterns of staining were not present in the corpus callosum for any of the groups. Therefore it can be concluded that repeated mTBI was not associated with widespread cell death or degeneration at this acute stage.

\section{Indicators of Structure, Reactivity and Inflammation in the CC}

Following single or repeated $\mathrm{mTBI}$ the $\mathrm{CC}$ was examined for evidence of changes in structure, or markers of reactivity or inflammation. There were no differences in the thickness of the posterior or middle $\mathrm{CC}$ with single or repeated mTBI (posterior $\mathrm{F}(3,24)=0.58$; middle $\mathrm{F}(3,24)$ $=1.045$, all $\mathrm{p}>0.05$, Figure 6A). Fine-scale analyses of node-paranode dimensions in the posterior body of the $\mathrm{CC}$ were conducted for a subset of animals, assessing tranverse sections 
and comparing dimensions of Caspr+ immunoreactivity in $\mathrm{N}=5$ animals per group. Assessments of node-paranode dimensions have been shown to reveal subtle differences in the structure of myelinated axons that reflect ultrastructural findings (Szymanski et al. 2013). There were no indications of changes in node-paranode structure with repeated mTBI when assessing: length of the paranodal gap, equating to length of the Node of Ranvier $(F(3,15)=$ $0.322, \mathrm{p}=0.81)$; node width $(\mathrm{F}(3,34)=0.954, \mathrm{p}=0.43)$; or length of the node-paranode complex, $(\mathrm{F}(3,15)=0.375, \mathrm{p}=0.77)($ Figure 6B) .

Immunofluorescence for GFAP, indicative of astrocyte reactivity, was observed throughout brain sections. There were no changes in GFAP immunointensity or area of staining in the anterior, middle or posterior CC, with single or repeated mTBI (statistics for immunointensity: anterior $F(3,24)=0.402, p>0.05$; middle $F(3,23)=0.406, p>0.05$; posterior $F(3,24)=0.462$, $p>0.05$, Figure 6C). Similary, there were no changes in IBA1+ immunointensity or area of staining in the same regions, indicating no changes in microglia numbers or area, when assessed following single or repeated mTBI over lambda on day 4 (statistics for immunointensity: anterior $\mathrm{F}(3,24)=0.704, \mathrm{p}>0.05$; middle $\mathrm{F}(3,24)=0.263, \mathrm{p}>0.05$; posterior $F(3,24)=1.769, p>0.05$, Figure 6D). Furthermore, there were no obvious indications of changes in IBA+ cell morphology to indicate activation of the microglia. The numbers of Olig2+/NG2+ OPCs were assessed in the CC and dentate gyrus, for the sham and $3 \mathrm{x}$ mTBI groups, in a single field of view. There were no significant differences in the posterior, middle or anterior CC or dentate gyrus of the hippocampus with 3x mTBI (sham vs. 3x mTBI Mean \pm SEM: posterior CC $3.33 \pm 0.96$ vs. $1.5 \pm 0.67$; middle CC $1.00 \pm 0.423$ vs. $1.33 \pm 0.62$; anterior CC $2.625 \pm 0.62$ vs. $1.33 \pm 0.67$; Dentate gyrus $2.125 \pm 0.52$ vs. $1.50 \pm 0.62$, all $\mathrm{p}>0.05$ ). 


\section{DISCUSSION}

Single and repeated closed-head mTBI over lambda in female rats produces a very mild behavioural phenotype, associated with no identifiable neuroanatomical or inflammatory changes in the first days after injury. Slightly more pronounced deficits are observed if the impact site is situated over bregma, but the increased incidence of hairline skull fractures and haemorrhage renders this impact location suboptimal to serve as a model of repeated mTBI. Our biomechanical measures of the forces imparted to the impact area are in line with values reported in the literature (Luo et al. 2014). We observed increased MBP immunoreactivity, similar to that found in areas vulnerable to secondary degeneration following neurotrauma (Fitzgerald et al. 2010), likely due to increased immunogenicity given that myelin debris was not observed (Kozlowski et al. 2008), and in line with similar studies assessing areas surrounding ischemia (Gregersen et al. 2001). An unexpected finding was reduced microglia levels in anterior regions of the cortex following injury, which is in contrast to the majority of the literature showing no change or increased microglia staining with mTBI (Fehily and Fitzgerald 2016) and may be due to death of cells in these particular regions, which were not assessed in the current study. However despite these changes, the high rate of skull fracture and haemorrhaging with bregma impact-sites indicated that using a lambda impact site is more suited to model repeated mTBI. To our knowledge this is the first demonstration in this closedhead mTBI model, that not only impact force, but also impact location, is an important determinant in injury severity and skull integrity, consistent with human literature (Pellman et al. 2003).

Our findings using the preferred model with mTBI impact over lambda, are in line with other studies of repeated mTBI using closed head injury models; behavioural and neuroanatomical deficits are subtle, particularly in the acute phase (Kane et al. 2012; Mannix et al. 2013). Our subtle acute changes in microglia are also in line with other studies using a closed head model 
of repeated mTBI, where microglial activation was not observed at 24 hours following $2 \mathrm{x}$ mTBI but had developed by day 7 after injury (Shitaka et al. 2011; Bennett et al. 2012). However, our observed acute increase in lipid peroxidation in a subset of neurons with increasing numbers of mTBI indicates oxidative damage is occurring in this model of repeated mTBI in female rats, and is likely to be associated with chronic deficits as pathology develops. Persistent and mild cognitive impairment are found in people with mTBI (Ramos-Zuniga et al. 2014) and some domains such as executive function are especially sensitive to the repetitive effects of mTBI (Karr et al. 2014). Similarly, we found a subtle yet robust change in MWM probe trial performance, but only following repeated mTBI. The decrease in time near the platform zone in the $2 \mathrm{x}$ mTBI group indicates a lack of preservation of spatial mapping, similar to Barnes maze deficits revealed in other closed-head mTBI models (Mouzon et al. 2012; Mouzon et al. 2014), and MWM deficits found in a model of mTBI where a projectile is fired at a helmet (Bolouri et al. 2012). These changes occurred despite lack of observed changes in the hippocampus for markers of apoptesis or-neurodegeneration, and may be a consequence of altered neurotransmission or connectivity. Nevertheless, increases in HNE positive neurons in the cortex were observed following $2 x$ mTBI, indicating damage had been initiated, and may spread as time passes after injury. MWM spatial memory impairments similar to those observed in the current study have occurred following TBI, in the absence of cell loss in the hippocampus (Lyeth et al. 1990).

A specific increase in the lipid peroxidation marker HNE was observed in cortical neurons, indicating oxidative stress in these cells as a consequence of repeated mTBI. As with behavioural changes when the impact site was over lambda, increased HNE+ neurons were only observed following $2 \mathrm{x}$ mTBI, suggesting that a single mTBI is not sufficient to increase HNE in this model. The lack of an acute effect on day 4 following $3 \mathrm{x}$ mTBI may reflect improvement relative to $2 \mathrm{x}$ mTBI insult, perhaps as a consequence of induction of endogenous 
antioxidant activity. Alternatively, reported biphasic production of superoxide in response to mTBI (Zhang et al. 2012) may indicate a transient decrease in oxidative stress and resultant transient rescue of cognitive deficit at day 4 in the $3 x$ mTBI group. Despite this, AlzPP increases were present following $3 \mathrm{x}$ mTBI, confirming the presence of continuing pathology. Future studies addressing longer term outcomes will be required to determine chronic consequences of increasing numbers of mTBI. Nevertheless, our data indicate significant complexities in outcomes following additional mTBI, when equal time is allowed for pathology to develop from an initial insult. Data from MWM in mice following mTBI (Meehan et al. 2012; Mannix et al. 2013) suggest that the length of time between repeated mTBI also has longterm effects on cognitive performance, with longer intervals reducing cognitive deficits. Further studies will be required to elucidate the effects of interval between mTBI, the number of mTBIs and the time after the last injury, on lipid peroxidation in neurons.

We have previously demonstrated increased areas of HNE+ immunoreactivity in optic nerve vulnerable to secondary degeneration following partial injury (O'Hare Doig et al. 2014), consistent with our findings of increased HNE+ cell numbers in the current study. Increased lipid peroxidation has been observed at 6,24 and 72 hours after more severe TBI, delivered by controlled cortical impact (Tyurin et al. 2000), with elevated HNE levels in the hippocampus at 96 hours (Ansari et al. 2008a). However, previous work which demonstrated changes in oxidative and nitrosative stress markers following repeated mTBI, used brain homogenates, precluding the ability to identify cell types (Tavazzi et al. 2007). To our knowledge, our study is the first to describe an increase in HNE+ neurons in the brain following repeated mTBI. HNE is both a product of lipid peroxidation and a toxic metabolite, serving as a sensitive indicator of oxidative damage to lipids. Other indicators of oxidative damage such as DNA oxidation were not altered, indicating that damage mechanisms are specific-and likely alse confined to particular cortical brain regions, at least in the acute stage. The focussed oxidative 
damage observed in the current study underlie the modest nature of the injury and are relevant to concussive and sub-concussive impacts, rather than other more severe TBI models. Indeed, the absence of changes in other oxidative stress indicators suggests that lipid peroxidation may play a particularly important role in neuropathological progression, at least acutely following injury. It is possible that antioxidant therapies may be of value in reducing early oxidative changes in repeated mTBI, as has been observed following more severe TBI in a range of models (Hall et al. 2010; Itoh et al. 2010). Co-localisation of HNE with the neuronal marker NeuN provides evidence that cells exhibiting lipid peroxidation following repeated mTBI were neuronal rather than glial. It is important to note that very few NeuN+ neurons were $\mathrm{HNE}+$ indicating that $\mathrm{HNE}+$ cells constitute a small proportion of the total neuronal pool, reflecting the mild disturbances in cognitive function observed. Thus it appears that not all neurons are equally affected by mTBI. Unlike previous studies assessing oxidative stress in secondary degeneration, we did not find evidence of HNE co-localisation with $\mathrm{CC} 1+$ oligodendrocytes, myelin basic protein or GFAP+ astrocytes (Wells et al. 2012; O'Hare Doig et al. 2014), indicating that repeated mTBI may activate oxidative stress pathways differently from other types of neurotrauma.

No gross neuroanatomical changes or indications of inflammation were observed in the current acute phase study in female rats when the impact was located over lambda. Changes in microglia, astrocytes and CC thickness have been observed at chronic time points ( $\geq 6$ months) following closed head mTBI (Mouzon et al. 2014), and acutely in more severe trauma (Loane and Byrnes 2010; Loane et al. 2014) or following a greater number of impacts (Mouzon et al. 2012), in male mice. Microglial activation increases over the week following closed head repeated mTBI, persisting in some brain regions (Shitaka et al. 2011); as such, increases in IBA1 immunoreactivity may be observed in female rats following repeated mTBI at later time points. Nevertheless, a study using a similar closed head repeated mTBI model to ours also 
failed to show differences in IBA1+ cells in the cortex, $\mathrm{CC}$, or hippocampus at 6 months in male mice, although changes in GFAP were present (Mannix et al. 2013), indicating that our understanding of developing pathology following repeated closed head mTBI is not yet complete. To our knowledge, the current study is the first to assess effects of repeated closed head mTBI using the 'Kimwipe' model in female rats, and provides important information regarding the mild nature of the acute injury phenotype in female rats using this model. The lack of changes in relatively sensitive measures of neurodegeneration such as Fluorojade and Caspase 3 -in hippocampus and CC confirm the mild nature of the injury. Note that we did not attempt to control for effects of the estrous cycle and as such, variability may have masked some alterations in outcome measures. Nevertheless, our data indicate that oxidative damage increases with repeated $\mathrm{mTBI}$ in female rats. The mild phenotype observed may be due to acute neuroprotective effects of oestrogen (Roof and Hall 2000). Further studies comparing both acute and chronic outcomes in male and female animals following repeated closed head mTBI will be required to elucidate gender specific long-term effects as the number of mTBI increases. In conclusion, this study of acute events following closed head mTBI identifies acute lipid peroxidation in neurons as a potential contributor to cognitive deficits. Our findings are consistent with those in more severe TBI in both animal and human studies (Signoretti et al. 2010; Hohl et al. 2012) and further indicate that lipid peroxidation may be a useful target for therapeutic intervention following concussion. 


\section{ACKNOWEDGEMENTS}

We acknowledge financial support from the Department of Health Western Australia Merit Award. MF is supported by an NHMRC Career Development Fellowship (APP1087114). We thank Dr Caitlin Wyrwoll for kindly allowing us to use her ANY-Maze software and Dr Jeremy Smith for providing selected antibodies.

\section{AUTHOR DISCLOSURE STATEMENT}

No competing financial interests exist. 


\section{REFERENCES}

Abdul-Muneer PM, Schuetz H, Wang F, et al. (2013) Induction of oxidative and nitrosative damage leads to cerebrovascular inflammation in an animal model of mild traumatic brain injury induced by primary blast. Free Radic Biol Med 60:282-291 doi: 10.1016/j.freeradbiomed.2013.02.029

Angoa-Pérez M, Kane MJ, Briggs DI, Herrera-Mundo N, Viano DC, Kuhn DM (2014) Animal models of sports-related head injury: bridging the gap between pre-clinical research and clinical reality. 129:916-931 doi: 10.1111/jnc.12690

Ansari MA, Roberts KN, Scheff SW (2008a) Oxidative stress and modification of synaptic proteins in hippocampus after traumatic brain injury. Free Radical Biology and Medicine 45:443-452 doi: http://dx.doi.org/10.1016/j.freeradbiomed.2008.04.038

Ansari MA, Roberts KN, Scheff SW (2008b) A time course of contusion-induced oxidative stress and synaptic proteins in cortex in a rat model of TBI. Journal of Neurotrauma 25:513-526 doi: 10.1089/neu.2007.0451

Bazarian JJ, Wong T, Harris M, Leahey N, Mookerjee S, Dombovy M (1999) Epidemiology and predictors of post-concussive syndrome after minor head injury in an emergency population. Brain Inj 13:173-189

Bennett RE, Mac Donald CL, Brody DL (2012) Diffusion tensor imaging detects axonal injury in a mouse model of repetitive closed-skull traumatic brain injury. Neurosci Lett 513:160-165 doi: 10.1016/j.neulet.2012.02.024

Binder LM (1997) A review of mild head trauma. part II: Clinical implications. Journal of Clinical and Experimental Neuropsychology 19:432-457 doi: $10.1080 / 01688639708403871$

Bolouri H, Saljo A, Viano DC, Hamberger A (2012) Animal model for sport-related concussion; ICP and cognitive function. Acta Neurol Scand 125:241-247 doi: 10.1111/j.1600-0404.2011.01614.x

Bolton AN, Saatman KE (2014) Regional neurodegeneration and gliosis are amplified by mild traumatic brain injury repeated at 24-hour intervals. J Neuropathol Exp Neurol 73:933-947 doi: 10.1097/NEN.0000000000000115

Broshek DK, Kaushik T, Freeman JR, Erlanger D, Webbe F, Barth JT (2005) Sex differences in outcome following sports-related concussion. Journal of Neurosurgery 102:856863

Buchele F, Morawska MM, Schreglmann SR, Penner M, Muser M, Baumann CR, Noain D (2016) Novel Rat Model of Weight Drop-Induced Closed Diffuse Traumatic Brain Injury Compatible with Electrophysiological Recordings of Vigilance States. J Neurotrauma 33:1171-1180 doi: 10.1089/neu.2015.4001

Cassidy JD, Carroll LJ, Peloso PM, et al. (2004) Incidence, risk factors and prevention of mild traumatic brain injury: results of the WHO Collaborating Centre Task Force on Mild Traumatic Brain Injury. Journal of rehabilitation medicine:28-60

Chen YUN, Constantini S, Trembovler V, Weinstock M, Shohami E (1996) An Experimental Model of Closed Head Injury in Mice: Pathophysiology, Histopathology, and Cognitive Deficits. Journal of Neurotrauma 13:557-568 doi: 10.1089/neu.1996.13.557

Cornelius C, Crupi R, Calabrese V, et al. (2013) Traumatic brain injury: Oxidative stress and neuroprotection. Antioxidants \& Redox Signaling 19:836-853 doi: 10.1089/ars.2012.4981

Dewitt DS, Perez-Polo R, Hulsebosch CE, Dash PK, Robertson CS (2013) Challenges in the development of rodent models of mild traumatic brain injury. Journal of Neurotrauma 30:688-701 doi: 10.1089/neu.2012.2349 
Fehily B, Fitzgerald M (2016) Repeated mild traumatic brain injury: potential mechanisms of damage. Cell Transplant doi: 10.3727/096368916X692807

Fitzgerald M, Bartlett CA, Harvey AR, Dunlop SA (2010) Early Events of Secondary Degeneration after Partial Optic Nerve Transection: An Immunohistochemical Study. Journal of Neurotrauma 27:439-452 doi: 10.1089/neu.2009.1112

Fuss B, Mallon B, Phan T, Ohlemeyer C, Kirchhoff F, Nishiyama A, Macklin WB (2000) Purification and Analysis of in Vivo-Differentiated Oligodendrocytes Expressing the Green Fluorescent Protein. Developmental Biology 218:259-274 doi: http://dx.doi.org/10.1006/dbio.1999.9574

Gao L, Macklin W, Gerson J, Miller RH (2006) Intrinsic and extrinsic inhibition of oligodendrocyte development by rat retina. Developmental Biology 290:277-286 doi: http://dx.doi.org/10.1016/j.ydbio.2005.11.007

Gregersen R, Christensen T, Lehrmann E, Diemer N, Finsen B (2001) Focal cerebral ischemia induces increased myelin basic protein and growth-associated protein- 43 gene transcription in peri-infarct areas in the rat brain. Experimental Brain Research 138:384-392 doi: 10.1007/s002210100715

Guskiewicz KM, Marshall SW, Bailes J, McCrea M, Cantu RC, Randolph C, Jordan BD (2005) Association between Recurrent Concussion and Late-Life Cognitive Impairment in Retired Professional Football Players. Neurosurgery 57:719-726 doi: 10.1227/01.neu.0000175725.75780.dd

Hall ED, Detloff MR, Johnson K, Kupina NC (2004) Peroxynitrite-mediated protein nitration and lipid peroxidation in a mouse model of traumatic brain injury. J Neurotrauma 21:9-20 doi: 10.1089/089771504772695904

Hall ED, Vaishnav RA, Mustafa AG (2010) Antioxidant therapies for traumatic brain injury. Neurotherapeutics 7:51-61 doi: 10.1016/j.nurt.2009.10.021

Higgins GC, Beart PM, Shin YS, Chen MJ, Cheung NS, Nagley P (2010) Oxidative stress: emerging mitochondrial and cellular themes and variations in neuronal injury. Journal of alzheimer's disease 20:453-473

Hohl A, da Silva Gullo J, Silva CCP, et al. (2012) Plasma levels of oxidative stress biomarkers and hospital mortality in severe head injury: a multivariate analysis. Journal of Critical Care 27:523-e511

Ito D, Imai Y, Ohsawa K, Nakajima K, Fukuuchi Y, Kohsaka S (1998) Microglia-specific localisation of a novel calcium binding protein, Iba1. Molecular Brain Research 57:19 doi: http://dx.doi.org/10.1016/S0169-328X(98)00040-0

Itoh T, Satou T, Nishida S, Tsubaki M, Imano M, Hashimoto S, Ito H (2010) Edaravone protects against apoptotic neuronal cell death and improves cerebral function after traumatic brain injury in rats. Neurochemical Research 35:348-355

Kane MJ, Angoa-Pérez M, Briggs DI, Viano DC, Kreipke CW, Kuhn DM (2012) A mouse model of human repetitive mild traumatic brain injury. Journal of Neuroscience Methods 203:41-49 doi: http://dx.doi.org/10.1016/j.jneumeth.2011.09.003

Karr JE, Areshenkoff CN, Garcia-Barrera MA (2014) The neuropsychological outcomes of concussion: a systematic review of meta-analyses on the cognitive sequelae of mild traumatic brain injury. Neuropsychology 28:321-336 doi: 10.1037/neu0000037

Kozlowski P, Raj D, Liu J, Lam C, Yung AC, Tetzlaff W (2008) Characterizing white matter damage in rat spinal cord with quantitative MRI and histology. J Neurotrauma 25:653-676

Kraus MF, Susmaras T, Caughlin BP, Walker CJ, Sweeney JA, Little DM (2007) White matter integrity and cognition in chronic traumatic brain injury: a diffusion tensor imaging study. Brain 130:2508-2519 doi: 10.1093/brain/awm216 
Loane DJ, Byrnes KR (2010) Role of Microglia in Neurotrauma. Neurotherapeutics 7:366377 doi: http://dx.doi.org/10.1016/j.nurt.2010.07.002

Loane DJ, Kumar A, Stoica BA, Cabatbat R, Faden AI (2014) Progressive neurodegeneration after experimental brain trauma: Association with chronic microglial activation. Journal of Neuropathology and Experimental Neurology 73:14-29

Longhi L, Saatman KE, Fujimoto S, et al. (2005) Temporal window of vulnerability to repetitive experimental concussive brain injury. Neurosurgery 56:364-374; discussion 364-374

Luo J, Nguyen A, Villeda S, et al. (2014) Long-term cognitive impairments and pathological alterations in a mouse model of repetitive mild traumatic brain injury. Frontiers in Neurology 5:12 doi: 10.3389/fneur.2014.00012

Lyeth BG, Jenkins LW, Hamm RJ, et al. (1990) Prolonged memory impairment in the absence of hippocampal cell death following traumatic brain injury in the rat. Brain Research 526:249-258 doi: http://dx.doi.org/10.1016/0006-8993(90)91229-A

Mannix R, Meehan WP, Mandeville J, et al. (2013) Clinical correlates in an experimental model of repetitive mild brain injury. Annals of Neurology 74:65-75 doi: 10.1002/ana. 23858

Meehan WPI, Zhang J, Mannix R, Whalen MJ (2012) Increasing Recovery Time Between Injuries Improves Cognitive Outcome After Repetitive Mild Concussive Brain Injuries in Mice. Neurosurgery 71:885-892 doi: 10.1227/NEU.0b013e318265a439

Morris R (1984) Developments of a water-maze procedure for studying spatial learning in the rat. Journal of Neuroscience Methods 11:47-60 doi: http://dx.doi.org/10.1016/01650270(84)90007-4

Mouzon B, Chaytow H, Crynen G, et al. (2012) Repetitive mild traumatic brain injury in a mouse model produces learning and memory deficits accompanied by histological changes. J Neurotrauma 29:2761-2773 doi: 10.1089/neu.2012.2498

Mouzon BC, Bachmeier C, Ferro A, et al. (2014) Chronic neuropathological and neurobehavioral changes in a repetitive mild traumatic brain injury model. Annals of Neurology 75:241-254 doi: 10.1002/ana.24064

Mullen RJ, Buck CR, Smith AM (1992) NeuN, a neuronal specific nuclear protein in vertebrates. Development 116:201-211

O'Hare Doig RL, Bartlett CA, Maghzal GJ, Lam M, Archer M, Stocker R, Fitzgerald M (2014) Reactive species and oxidative stress in optic nerve vulnerable to secondary degeneration. Exp Neurol 261C:136-146 doi: 10.1016/j.expneurol.2014.06.007

Payne SC, Bartlett CA, Savigni DL, Harvey AR, Dunlop SA, Fitzgerald M (2013) Early Proliferation Does Not Prevent the Loss of Oligodendrocyte Progenitor Cells during the Chronic Phase of Secondary Degeneration in a CNS White Matter Tract. Plos One 8 doi: e6571010.1371/journal.pone.0065710

Pellman EJ, Viano DC, Tucker AM, Casson IR (2003) Concussion in Professional Football: Location and Direction of Helmet Impacts-Part 2. Neurosurgery 53:1328-1341

Pratico D, Reiss P, Tang LX, Sung S, Rokach J, McIntosh TK (2002) Local and systemic increase in lipid peroxidation after moderate experimental traumatic brain injury. Journal of Neurochemistry 80:894-898

Psachoulia K, Jamen F, Young KM, Richardson WD (2009) Cell cycle dynamics of NG2 cells in the postnatal and ageing brain. Neuron Glia Biology 5:57-67 doi: doi:10.1017/S1740925X09990354

Rabadi MH, Jordan BD (2001) The Cumulative Effect of Repetitive Concussion in Sports. Clinical Journal of Sport Medicine 11:194-198 
Ramos-Zuniga R, Gonzalez-de La Torre M, Jimenez-Maldonado M, et al. (2014) Postconcussion Syndrome and Mild Head Injury: The Role of Early Diagnosis Using Neuropsychological Tests and Functional Magnetic Resonance/Spectroscopy. World Neurosurgery 82:828-835 doi: 10.1016/j.wneu.2013.09.018

Roof RL, Hall ED (2000) Estrogen-related gender difference in survival rate and cortical blood flow after impact-acceleration head injury in rats. J Neurotrauma 17:1155-1169 doi: 10.1089/neu.2000.17.1155

Saunders NR, Dziegielewska KM, Mollgard K, Habgood MD (2015) Markers for blood-brain barrier integrity: how appropriate is Evans blue in the twenty-first century and what are the alternatives? Front Neurosci 9:385 doi: 10.3389/fnins.2015.00385

Schneider C, Porter NA, Brash AR (2008) Routes to 4-Hydroxynonenal: Fundamental Issues in the Mechanisms of Lipid Peroxidation. Journal of Biological Chemistry 283:15539-15543 doi: 10.1074/jbc.R800001200

Shitaka Y, Tran HT, Bennett RE, Sanchez L, Levy MA, Dikranian K, Brody DL (2011) Repetitive closed-skull traumatic brain injury in mice causes persistent multifocal axonal injury and microglial reactivity. J Neuropathol Exp Neurol 70:551-567 doi: 10.1097/NEN.0b013e31821f891f

Signoretti S, Vagnozzi R, Tavazzi B, Lazzarino G (2010) Biochemical and neurochemical sequelae following mild traumatic brain injury: summary of experimental data and clinical implications. Neurosurgical Focus 29:1-12 doi: 10.3171/2010.9.FOCUS10183

Singh IN, Sullivan PG, Deng Y, Mbye LH, Hall ED (2006) Time course of post-traumatic mitochondrial oxidative damage and dysfunction in a mouse model of focal traumatic brain injury: implications for neuroprotective therapy. Journal of Cerebral Blood Flow \& Metabolism 26:1407-1418 doi: 10.1038/sj.jcbfm.9600297

Smith SL, Andrus PK, Zhang JR, Hall ED (1994) Direct measurement of hydroxyl radicals, lipid peroxidation, and blood-brain barrier disruption following unilateral cortical impact head injury in the rat. J Neurotrauma 11:393-404 doi: 10.1089/neu.1994.11.393

Stahel PF, Shohami E, Younis FM, et al. (2000) Experimental Closed Head Injury[colon] Analysis of Neurological Outcome, Blood-Brain Barrier Dysfunction, Intracranial Neutrophil Infiltration, and Neuronal Cell Death in Mice Deficient in Genes for ProInflammatory Cytokines. J Cereb Blood Flow Metab 20:369-380

Szymanski CR, Chiha W, Morellini N, et al. (2013) Paranode abnormalities and oxidative stress in optic nerve vulnerable to secondary degeneration: modulation by $670 \mathrm{~nm}$ light treatment. PLoS One 8:e66448 doi: 10.1371/journal.pone.0066448 PONE-D-1308628 [pii]

Tavazzi B, Vagnozzi R, Signoretti S, et al. (2007) Temporal window of metabolic brain vulnerability to concussions: oxidative and nitrosative stresses-- part II. Neurosurgery 61:390-396

Tyurin VA, Tyurina YY, Borisenko GG, et al. (2000) Oxidative Stress Following Traumatic Brain Injury in Rats. Journal of Neurochemistry 75:2178-2189 doi: 10.1046/j.14714159.2000.0752178.x

Vagnozzi R, Tavazzi B, Signoretti S, et al. (2007) Temporal window of metabolic brain vulnerability to concussions: mitochondrial-related impairment - part I. Neurosurgery 61:379-389 doi: 10.1227/01.NEU.0000280002.41696.D8

Valavanidis A, Vlachogianni T, Fiotakis C (2009) 8-hydroxy-2' -deoxyguanosine (8-OHdG): A Critical Biomarker of Oxidative Stress and Carcinogenesis. Journal of Environmental Science and Health, Part C 27:120-139 doi: $10.1080 / 10590500902885684$ 
Wells J, Kilburn MR, Shaw JA, Bartlett CA, Harvey AR, Dunlop SA, Fitzgerald M (2012) Early in vivo changes in calcium ions, oxidative stress markers, and ion channel immunoreactivity following partial injury to the optic nerve. Journal of Neuroscience Research 90:606-618 doi: 10.1002/jnr.22784

Werner C, Engelhard K (2007) Pathophysiology of traumatic brain injury. British Journal of Anaesthesia 99:4-9 doi: 10.1093/bja/aem131

Yamada KH, Kozlowski DA, Seidl SE, et al. (2012) Targeted gene inactivation of calpain-1 suppresses cortical degeneration due to traumatic brain injury and neuronal apoptosis induced by oxidative stress. The Journal of Biological Chemistry 287:13182-13193 doi: 10.1074/jbc.M111.302612

Zhang QG, Laird MD, Han D, et al. (2012) Critical role of NADPH oxidase in neuronal oxidative damage and microglia activation following traumatic brain injury. PLoS One 7:e34504 doi: 10.1371/journal.pone.0034504 


\section{Figure Legends}

Figure 1. A) Images showing the mTBI apparatus and 3 infra-red cameras and B) animal positioning under guide tube in weight-drop device. C) Schematic illustrating lambda groundtruth and trial impact sites $(\mathrm{N}=3)$ and $\mathrm{D})$ bregma ground-truth and trial impact sites $(\mathrm{N}=3)$.

Figure 2. Blood brain barrier breaches, IBA1, MBP and HNE immunoreactivity in cortex following sham or 3x mTBI located over bregma or lambda. A) Median, interquartile range and range blood brain barrier breach score on days 3 and 4, with B) representative images of scoring system employed. C) The median, interquartile range and range number of HNE+ cells in coronal sections of the cortex on days 3 and 4. D) Distribution of HNE+ cells in a representative coronal section of the cortex; the yellow dots indicate HNE+ cells. E) Median, interquartile range and range IBA1+ cells in posterior, middle and anterior cortex regions on days 3 and 4, with F) representative images from anterior cortex. The inset in the leftmost panel shows magnified image of IBA1+ cells in the sham animal. G) Median, interquartile range and range MBP immunoreactivity in posterior, middle and anterior cortical regions as well as in $\mathrm{CC}$ on day 3, with $\mathrm{H}$ ) representative images showing areas of analysis (yellow dotted line region). Significant differences are indicated $* \mathrm{p} \leq 0.05$

Figure 3. Behavioural outcomes following sham, 1x, $2 \mathrm{x}$ or $3 \mathrm{x}$ mTBI on days $1-4 . \mathrm{A})$ The median, interquartile range and range for total NSS are shown for each day of testing, with proportion foot faults on the $1 \mathrm{~cm}$ beam in B). C) Median, interquartile range and range time to reach the platform in the MWM task following sham, $1 \mathrm{x}, 2 \mathrm{x}$ or $3 \mathrm{x}$ mTBI on days 1 to 3 . The non-striped histogram-bars show the average of the first set of trials, the striped histogram bars show the average of the second set of trials. The median, interquartile range and range Mean + SEM: D) time spent in the opposite quadrant to the original platform location; E) in the 
quadrant which previously contained the platform; F) distance from the previous platform location; during the probe trial, for each group on day 4. G) Regression plot and correlation between the number of mTBI received and the mean distance from the platform on the probe trials. The solid line shows the regression line of best fit and the dotted lines show the 95\% confidence intervals. Post-hoc statistical significance for all quantifications: ${ }^{*} \mathrm{p} \leq 0.05$.

Figure 4. 4-Hydroxynoneonal (HNE) and cell identification marker immunoreactivity in the cortex following sham, $1 \mathrm{x}, 2 \mathrm{x}$ or $3 \mathrm{x}$ mTBI on day 4. A) Distribution of HNE+ cells from a representative sagittal section of the cortex; the white dots indicate HNE+ cells. The horizontal dotted lines delineate the regions used for quantification. B) The median number of HNE+ cells in photomontaged sagittal sections of the cortex with the interquartile range and range, significant differences are indicated $* \mathrm{p} \leq 0.05$. HNE+ cells were not immunopositive for NG2 (C), CC1 or Olig2 (D), IBA1 (E) or GFAP (F) but were immunopositive for neuronal marker NeuN (F) using z-stack images and confocal microscopy. HNE+ cells indicated by arrowheads, with co-localisation with cell specific markers indicated by arrows (teal nuclei indicates NeuN and Hoechst co-localisation, surrounded by red HNE). Scale bars for each line of images are as shown.

Figure 5. Oxidative stress and Caspase $3 \underline{\mathrm{AlzPP}}$ immunoreactivity in the brain following sham, $1 \mathrm{x}, 2 \mathrm{x}$ or $3 \mathrm{x}$ mTBI on day 4. A) Median, interquartile range and range Mean \pm SEM immunofluorescence intensity of 8OHDG in selected regions of cortex. Representative images are taken from the posterior cortex. B) Median and interquartile range of MnSOD immunopositive cells in the dentate gyrus of the hippocampus, with representative images. C) Mean \pm SEM Caspase 3+ cell numbers in selected regions of the CC and hippocampus, with 
representative images from the posterior $\mathrm{CC}$. Scale bars for all images are as shown. D) Representative images of AlzPP immunoreactivity (green) in middle cortex following sham or 3x mTBI on day 4, together with Hoechst nuclear stain; immunopositive cells are identified with arrows; note the lower immunoreactivity in sham.

Figure 6. Neuroanatomical, structural and cellular measures in the CC following sham, 1x, 2x or 3x mTBI on day 4; all bar graphs show median, interquartile range and range mean \pm SEM. A) CC thickness anteriorly (genu) and posteriorly (splenium) in Luxol fast blue and Cresyl violet stained tissue. Example images are from the posterior CC. B) Quantification of nodeparanode structure in the posterior (splenium) $\mathrm{CC}$. The example image shows the measures used in the analysis, corresponding to the letters on the graph above the histogram bars. C) Quantification of GFAP immunointensity in the CC, with representative images from the posterior CC. D) Quantification of IBA1 immunointensity in the CC, with representative images from the posterior CC. No significant differences were observed for any measure ( $\mathrm{p}>$ $0.05)$. 


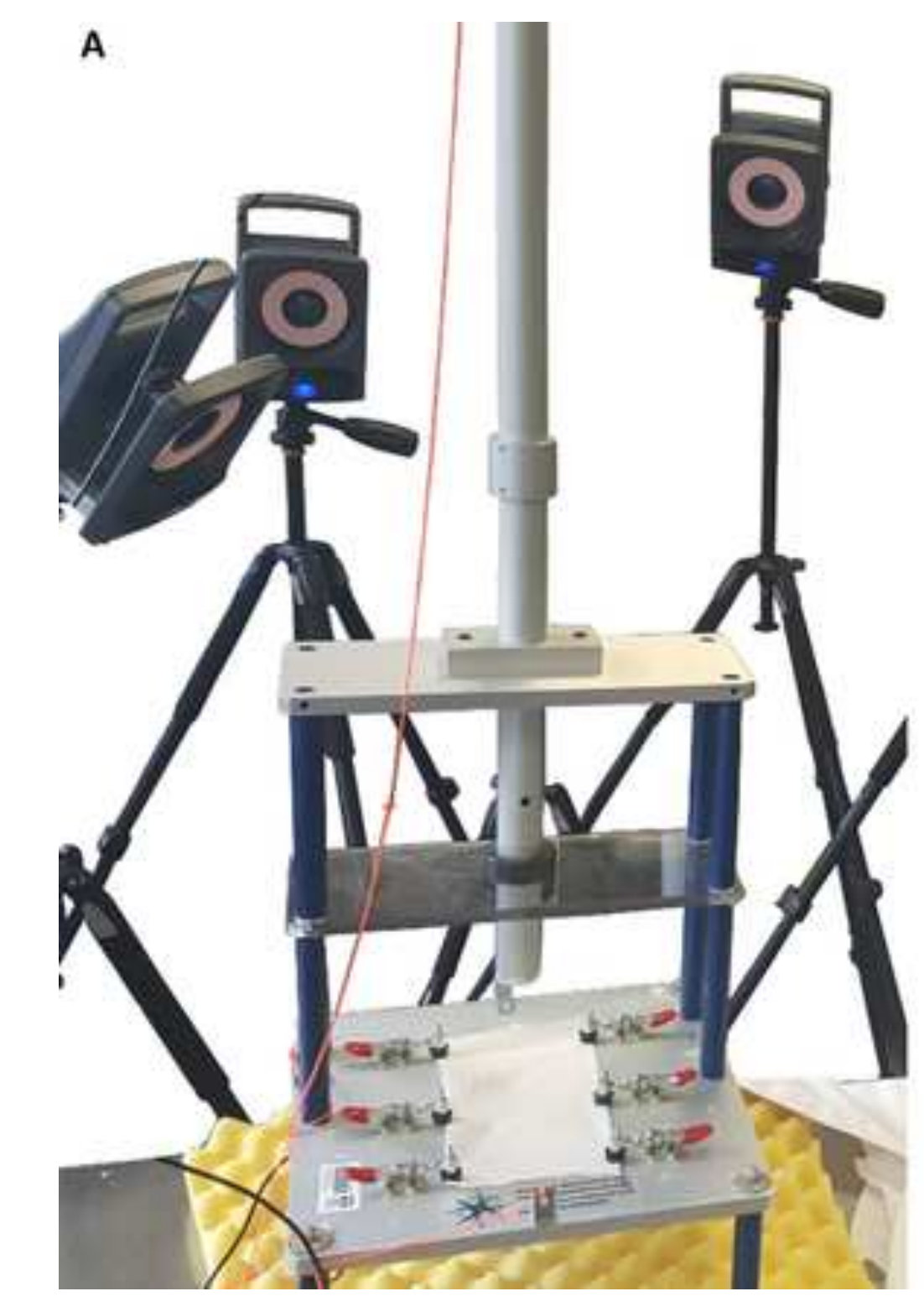

B

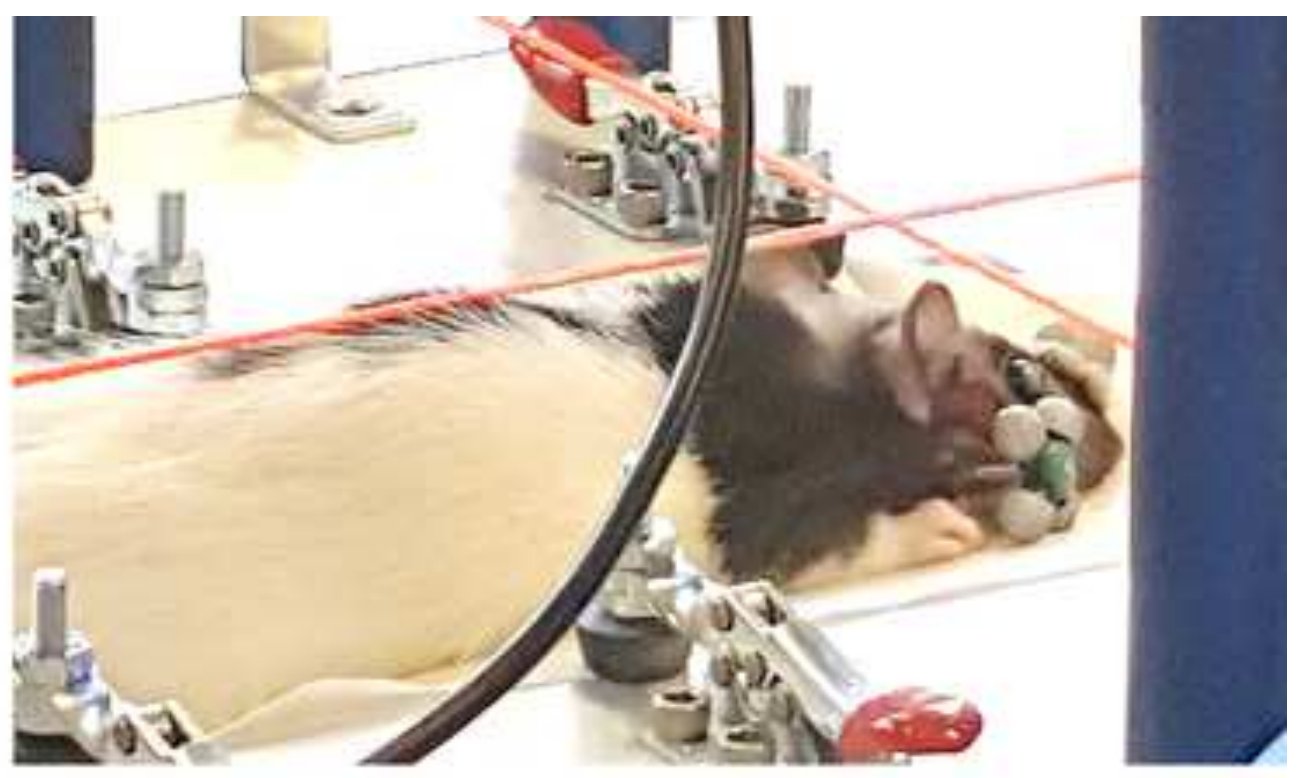

C

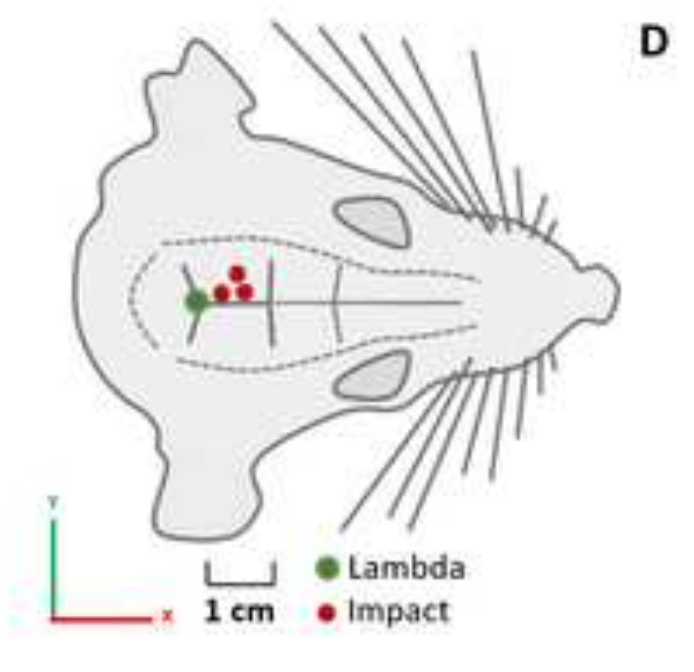

D

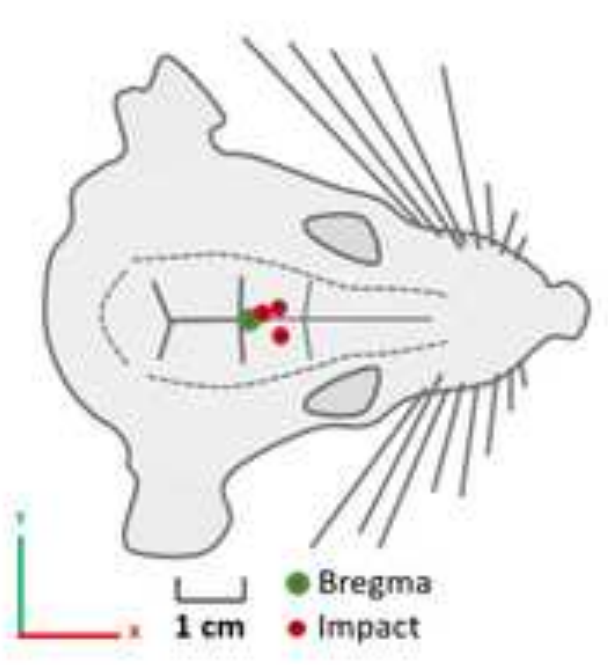

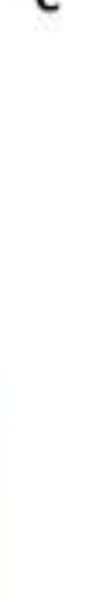


A Blood Brain Barrier

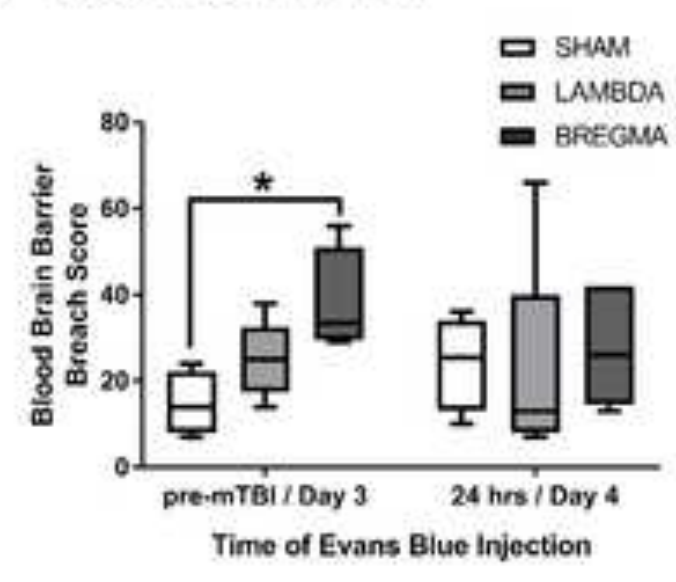

B

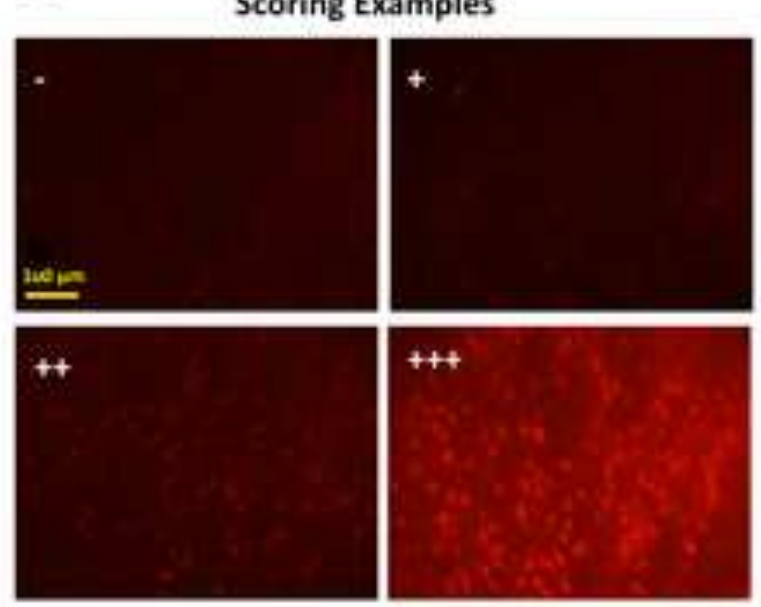

E
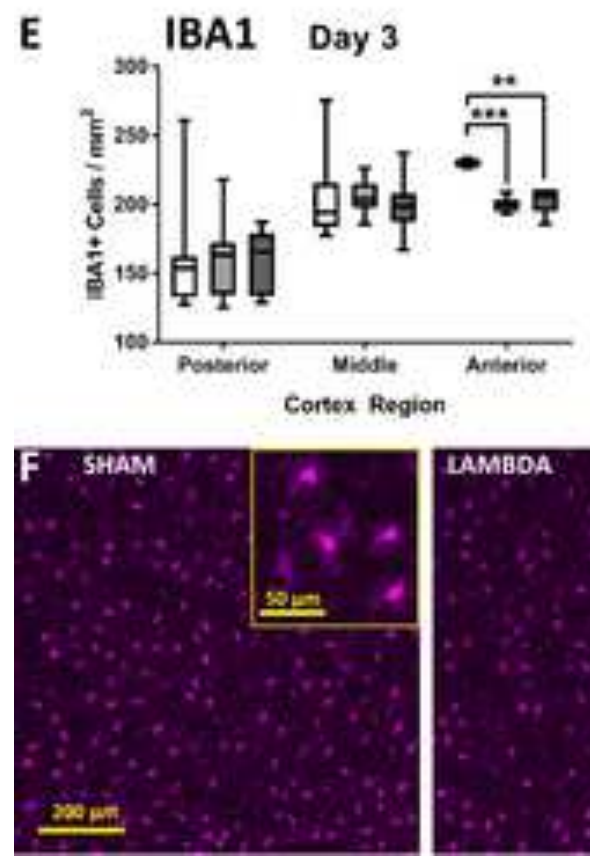

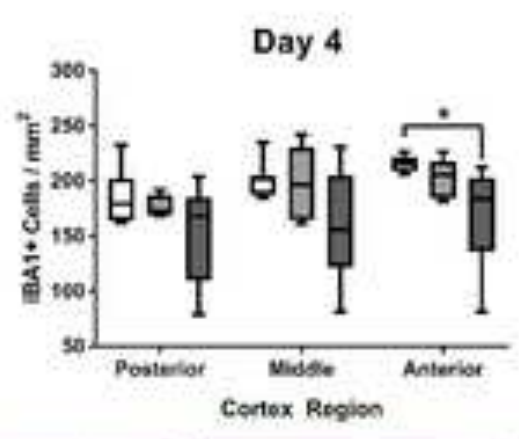

BAEGMA
G MBP

C HNE Cells in Cortex
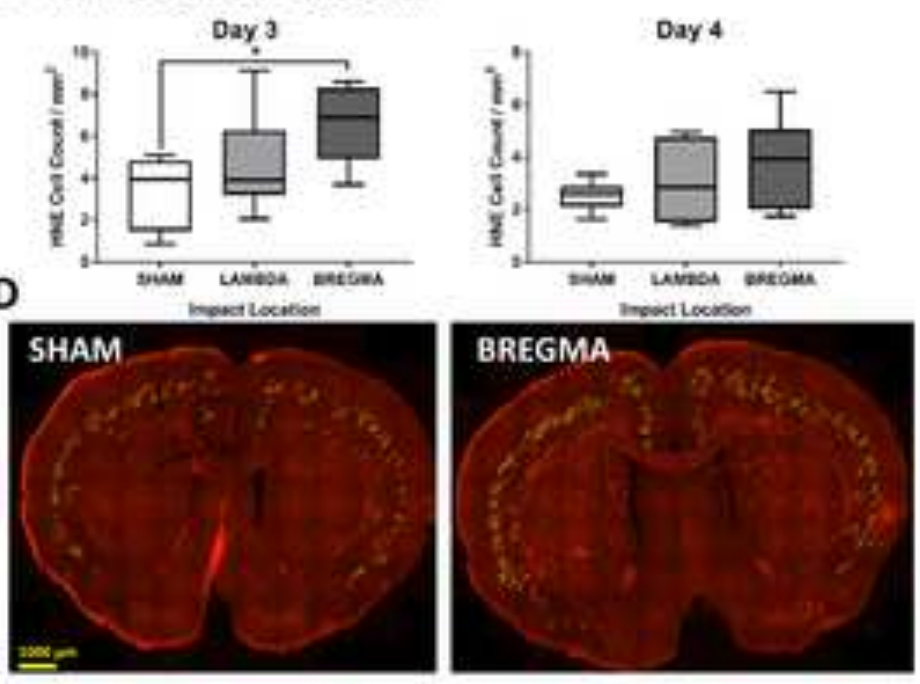

Day 3
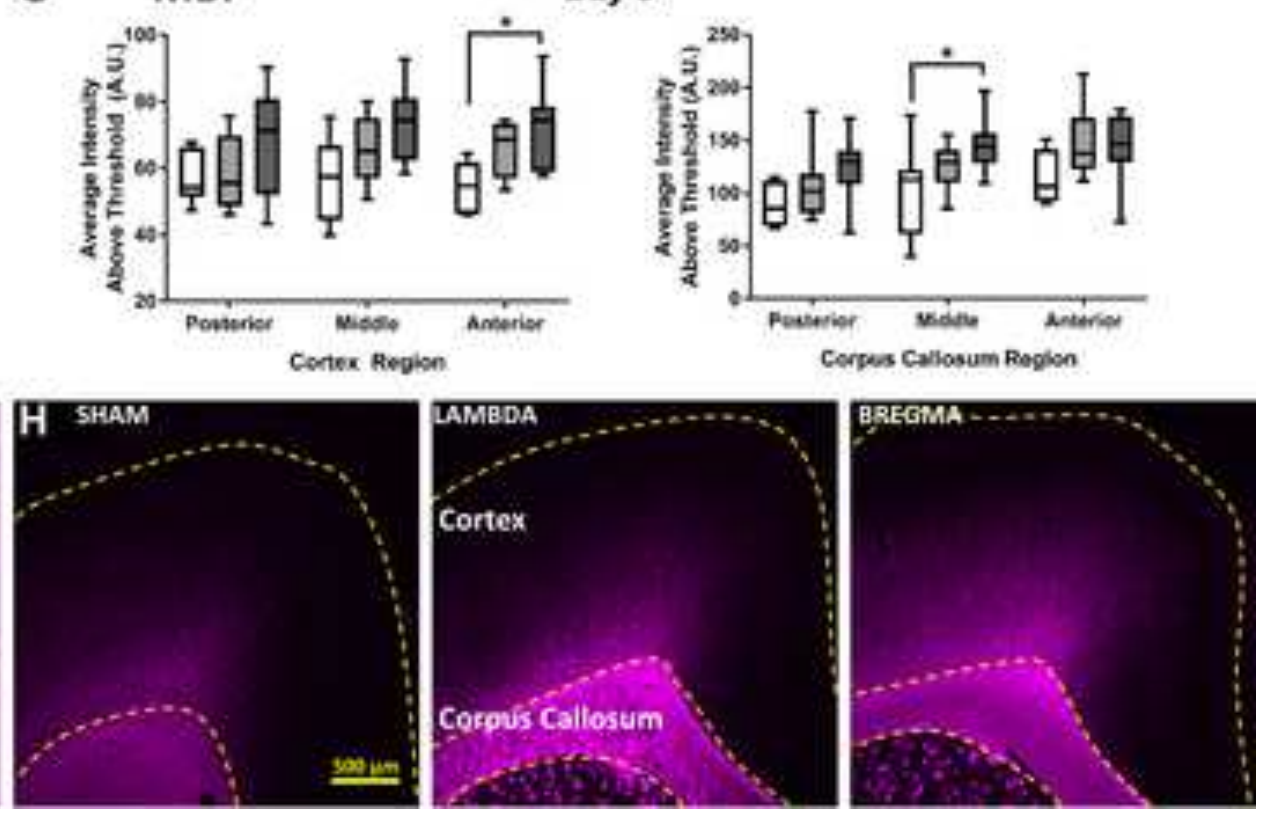

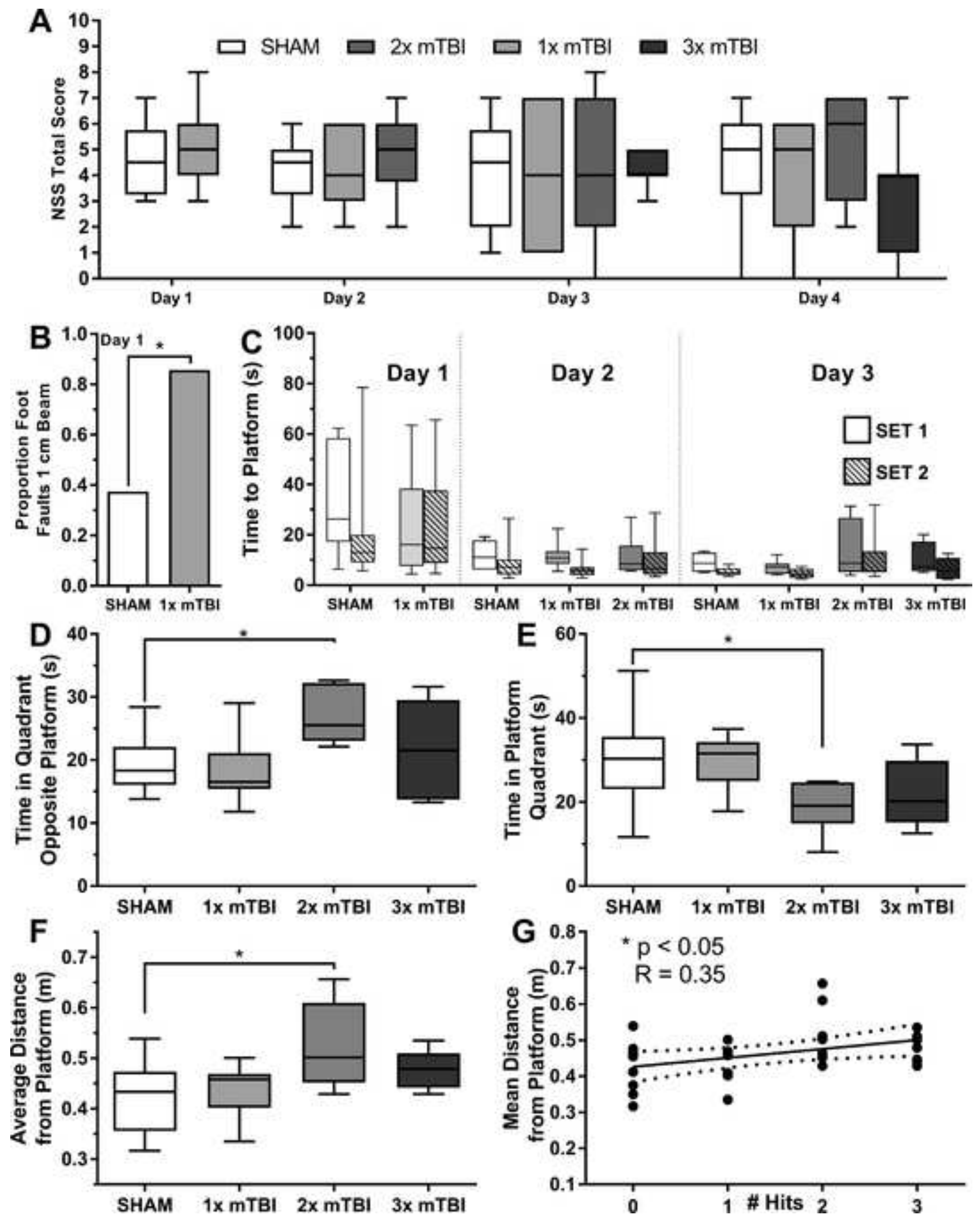


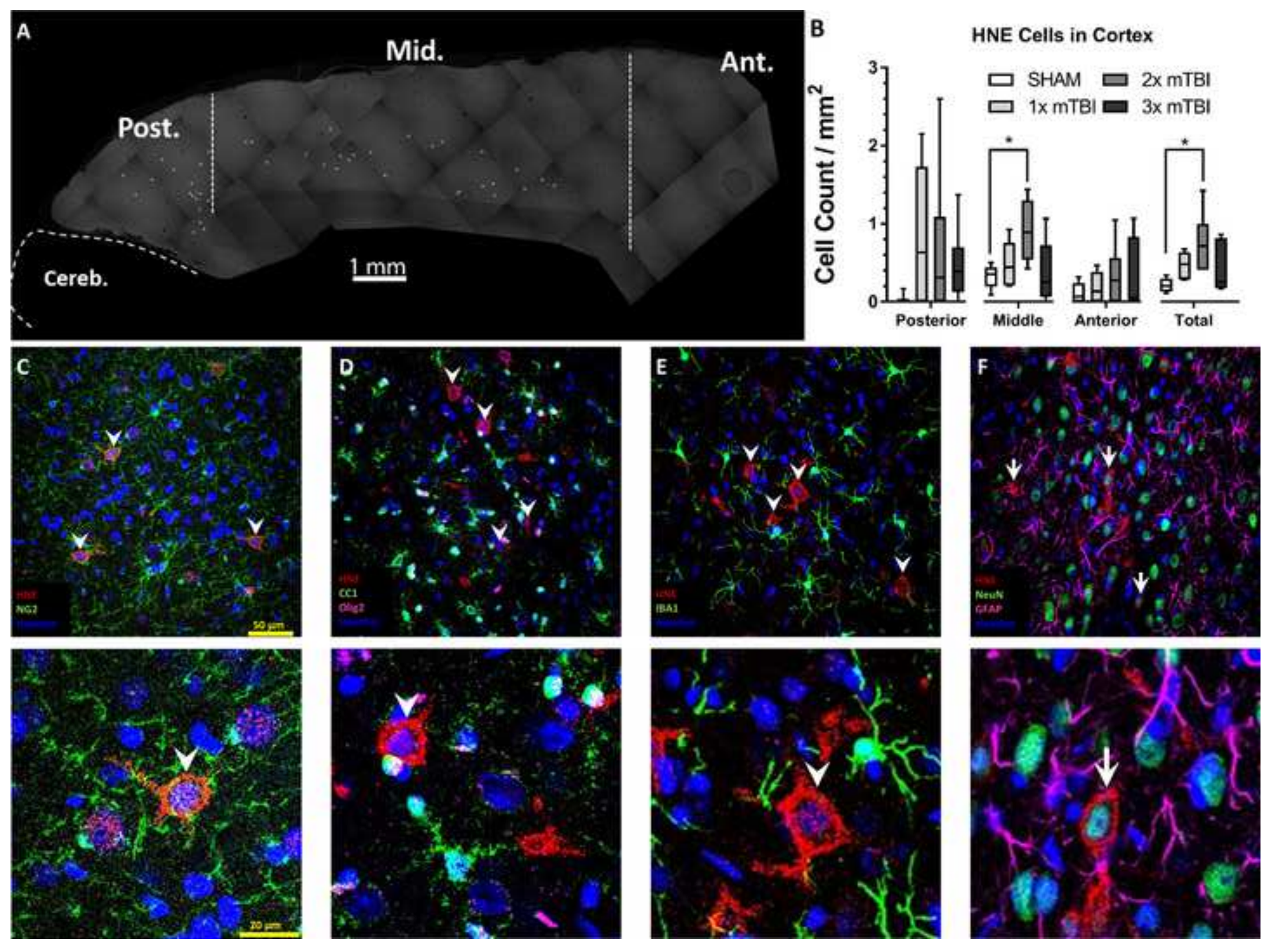




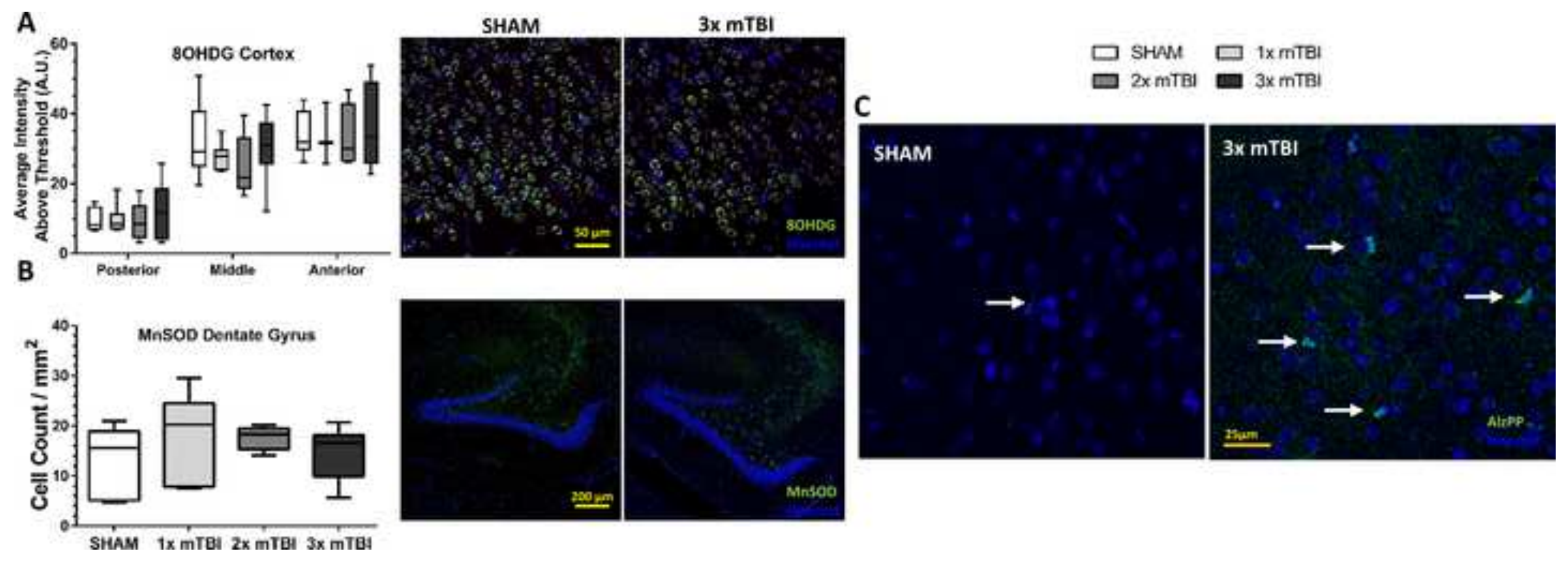




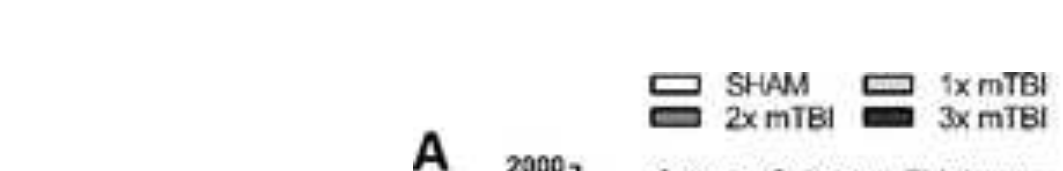

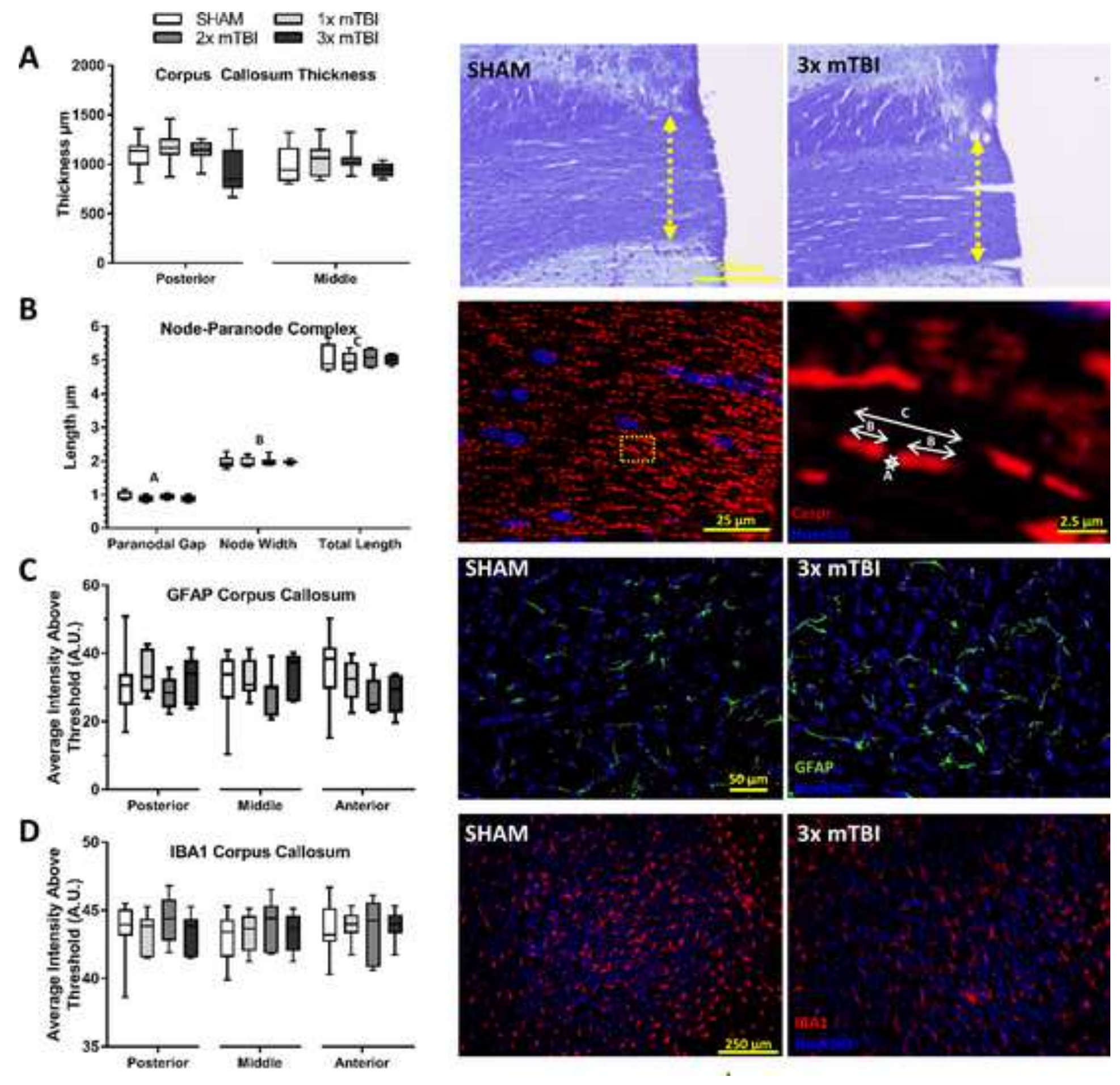

\section{A}

B 
Click here to access/download Supplementary Material Bregma v Lamda Comparison.avi 